\title{
Holistic face processing is influenced by non-conscious visual information
}

Short title: non-conscious holistic face processing

Haiyang Jin ${ }^{1}$, Matt Oxner $^{2}$, Paul M. Corballis ${ }^{1}$, William G. Hayward ${ }^{3 *}$

1 School of Psychology, University of Auckland, New Zealand

2 Institute of Psychology, University of Leipzig, Leipzig, Germany

3 Department of Psychology, University of Hong Kong, Hong Kong

*Corresponding author information:

Prof. William G. Hayward, Department of Psychology, The University of Hong Kong, Hong Kong (e-mail: whayward@hku.hk).

This work has been accepted in British Journal of Psychology. Its content may be different from the final version. https://doi.org/10.1111/bjop.12521 


\title{
Author contributions
}

Haiyang Jin: conceptualization, data curation, formal analysis, investigation, funding acquisition, methodology, project administration, resources, software, visualization, writing original draft, writing - review \& editing

Matt Oxner: data curation, methodology, software, writing - review \& editing

Paul M. Corballis: supervision, writing - review \& editing

William G. Hayward: conceptualization, funding acquisition, investigation, supervision, writing - review \& editing

\begin{abstract}
Holistic face processing has been widely implicated in conscious face perception. Yet, little is known about whether holistic face processing occurs when faces are processed unconsciously. The present study used the composite face task and continuous flash suppression (CFS) to inspect whether the processing of target facial information (the top half of a face) is influenced by irrelevant information (the bottom half) that is presented unconsciously. Results of multiple experiments showed that the composite effect was observed in both the monocular and CFS conditions, providing the first evidence that the processing of top facial halves is influenced by the aligned bottom halves no matter whether they are presented consciously or unconsciously. However, much of the composite effect for faces without masking was disrupted when bottom facial parts were rendered with CFS. These results suggest that holistic face processing can occur unconsciously, but also highlight the significance of holistic processing of consciously presented faces.
\end{abstract}


Key words: face perception; holistic processing; subliminal; preconscious; composite face task; continuous flash suppression

\section{Data availability statement}

The data that support the findings of this study are openly available in Open Science Framework at https://osf.io/rwv6f/.

\section{Acknowledgements}

This work was funded by a grant (HKU17608519) from the General Research Fund of the Hong Kong Research Grants Council to William G. Hayward, and co-funded by a China Scholarship Council (CSC) grant ([2015]08330295) awarded to Haiyang Jin. 


\section{Introduction}

Humans are believed to be good at processing faces (Duchaine \& Nakayama, 2006; Rossion, 2018; Rossion \& Michel, 2018; Young \& Burton, 2018a, 2018b). Underlying this extraordinary skill, many researchers have claimed that faces are processed in a holistic manner (e.g., Hole, 1994; Young, Hellawell, \& Hay, 1987), which means that people are inclined to process the multiple facial parts as a perceptual whole or gestalt (Hayward, Crookes, \& Rhodes, 2013; Maurer, Grand, \& Mondloch, 2002; Rezlescu, Susilo, Wilmer, \& Caramazza, 2017; Rossion, 2008, 2013). This hypothesis is supported by a number of experimental paradigms. For instance, the part-whole effect demonstrates that recognition of a target feature is facilitated by other facial information (Tanaka \& Farah, 1993; Tanaka \& Simonyi, 2016). The composite face illusion is found when the same top facial halves are perceived as different when they are aligned with distinct bottom halves (Rossion, 2013; Young et al., 1987). More generally, the processing of one kind of facial information is also affected by other (e.g., Amishav \& Kimchi, 2010; Hayward, Crookes, Chu, Favelle, \& Rhodes, 2016; Meinhardt-Injac, Persike, \& Meinhardt, 2010, 2014; Tanaka \& Sengco, 1997). These findings suggest that various types of facial information are obligatorily integrated into a holistic representation for processing faces.

Many studies have been conducted to address various aspects of holistic face processing in the last three decades (for reviews, Richler \& Gauthier, 2014; Rossion, 2013). It is worth noting that almost all these studies only concentrated on the holistic processing of conscious faces and affirmed that conscious faces could be processed holistically (e.g., Hayward et al., 2016; Wang et al., 2019). However, less is known about the processing of faces presented unconsciously. 
Studying the unconscious processing of faces has the potential to enhance our understanding of several different domains (Axelrod, Bar, \& Rees, 2015). On the one hand, various cognitive systems involved in face processing and the related face-selective neural indicators enable faces to serve as an excellent domain to investigate the general mechanisms underlying unconscious processing. On the other hand, taking an unconscious approach to face processing can also resolve some of the key issues and major debates in the field. Demonstrations of unconscious processing of facial information have been found for facial expressions (Jiang \& He, 2006; Jiang et al., 2009; Kiss \& Eimer, 2008; Williams, Morris, McGlone, Abbott, \& Mattingley, 2004; for reviews, Pessoa, 2005; Tamietto \& de Gelder, 2010), familiarity (de Gardelle \& Kouider, 2010; Henson, Mouchlianitis, Matthews, \& Kouider, 2008; Kouider, Eger, Dolan, \& Henson, 2009), gender (Finkbeiner \& Palermo, 2009; Khalid, Finkbeiner, König, \& Ansorge, 2013; Quek \& Finkbeiner, 2014; but see Amihai, Deouell, \& Bentin, 2011), gaze (Sato, Okada, \& Toichi, 2007; Stein, Peelen, \& Sterzer, 2012; Yokoyama, Noguchi, \& Kita, 2013), and shape (Stein \& Sterzer, 2011). By contrast, visual awareness appears to be a prerequisite for face identity (Moradi, Koch, \& Shimojo, 2005) and ethnicity (Amihai et al., 2011).

To sum up the two streams of literature, it is suggested that conscious faces could be processed holistically, and some facial information could be processed unconsciously, which leads to the question: can unconscious faces be processed holistically? On the one hand, holistic face processing probably could be executed without conscious awareness. First, holistic processing, indexed by the composite effect, was stronger for low spatial frequency information (Goffaux, 2009; Goffaux \& Rossion, 2006; but see Cheung, Richler, Palmeri, \& Gauthier, 2008), which has been shown consistently to be processed unconsciously (de Gardelle \& Kouider, 2010; Khalid et al., 2013). Second, the automaticity of holistic processing has been suggested as the results of perceptual expertise (Richler, Wong, \& 
Gauthier, 2011). Since one of the characteristics of automatic processing is to be able to execute unconsciously (Posner \& Snyder, 1975), perhaps holistic face processing could also be carried out unconsciously. In addition, Stein \& Sterzer (2011) manipulated the shapes of faces by contracting and expanding the internal facial information (i.e., features and configurations between them), and found evidence for unconscious processing. As consciously presented face shapes underlie composite effects (Jiang, Blanz, \& Rossion, 2011), and consciously presented irrelevant facial information (e.g., configurations) alters the processing of target information (e.g., features) as well (Amishav \& Kimchi, 2010; Richler, Palmeri, \& Gauthier, 2015; Tanaka \& Sengco, 1997), this unconscious processing of face shapes might also influence the processing of other facial information. Further, this opens the possibility that holistic face processing could occur unconsciously.

On the other hand, maybe holistic face processing depends on seeing faces. A previous study suggested that visual awareness was necessary for processing face identity (Moradi et al., 2005). Considering the significant role of holistic processing in face processing, it might be the necessity of conscious awareness for holistic processing that causes face identity judgments to require conscious awareness.

One previous study explored whether unconscious faces can be processed holistically (Axelrod \& Rees, 2014). They found that the processing of target parts was interfered with by the irrelevant parts only in the conscious, but not unconscious, condition, suggesting the necessity of conscious awareness for holistic face processing. However, there are several reasons to consider this result more critically. First, the design used by Axelrod and Rees (2014) might be underpowered for detecting the holistic processing of unconscious faces, since the sample sizes in both experiments were small (less than nineteen participants). Second, the dependent variable in their paradigms, i.e., accuracy, did not seem to be reliable. In addition to being easily subjected to response bias (as shown by Richler, Cheung, \& 


\section{same}

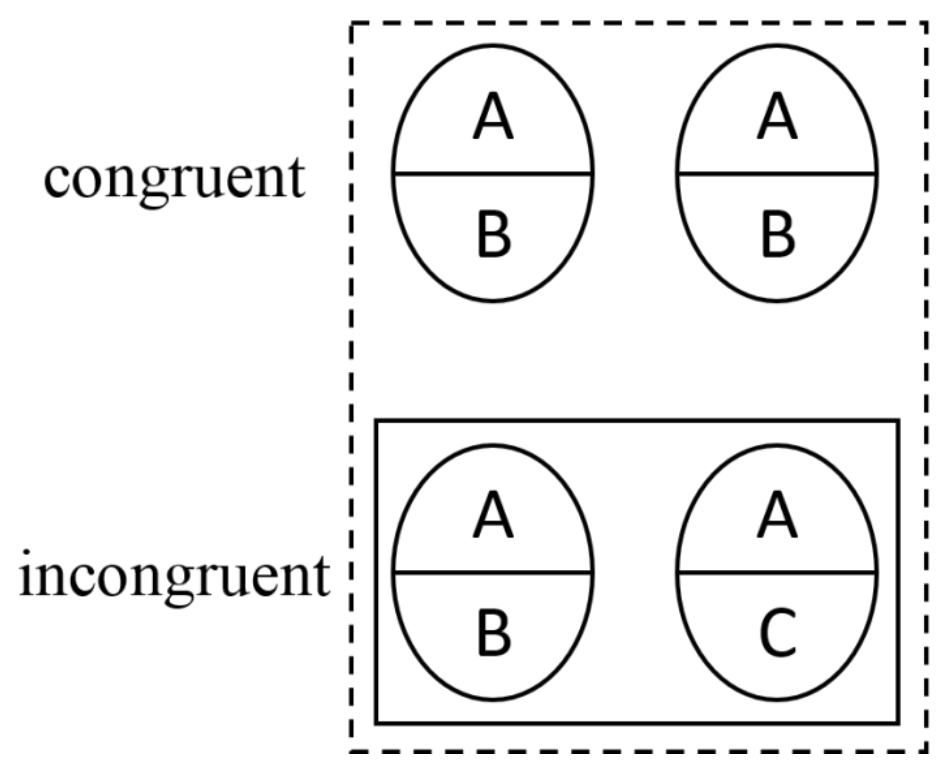

different
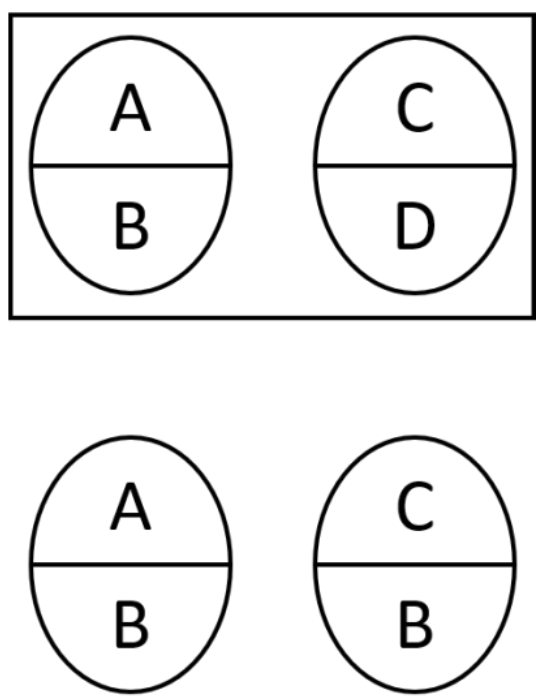

Figure 1. The experimental designs of the standard and complete composite tasks and the design used in Axelrod and Rees (2014). Here is the example when the top halves are used as the target and only the aligned composite faces are displayed. The two rows display the two levels of Congruency (congruent vs. incongruent) and two columns displays the two levels of SameDifferent (same vs. different). Different letters denote different facial identities. (1) The complete design consists of all the four conditions. (2) The standard design includes the same incongruent and different congruent conditions, as shown in the two solid line squares. (3) The dashed line square describes the conditions used in Axelrod and Rees (2014), in which they adopted eyes, instead of top halves, as the target.

Gauthier, 2011), accuracy is also vulnerable to guessing when the target parts were always the same, which was the case in their experiments. Moreover, the paradigm utilized by Axelrod and Rees (2014) was different from both of the popular composite face paradigms, i.e., the standard (Rossion, 2013) and complete (Richler \& Gauthier, 2014; Richler, Palmeri, \& Gauthier, 2012) designs (Figure 1). Considering the controversy about paradigms to measure holistic face processing (e.g., Richler \& Gauthier, 2013, 2014; Richler et al., 2012; Rossion, 2013), applying yet another paradigm is not ideal.

In the present study, we revisit the role of consciousness in holistic face processing. More specifically, the composite face task was used to measure holistic face processing, and continuous flash suppression (CFS) was used to mask presentation of part of the face composite, with the intention of presenting that part unconsciously. 
There are two popular paradigms for the composite face task (Figure 1): the standard design (Rossion, 2013) and the complete design (Richler \& Gauthier, 2014). Both paradigms examine the influence of irrelevant facial parts on target parts. One distinction between these two paradigms is that in the standard design, whereas the top halves of composite faces can be the same or different, the bottom halves are always different, as showed in the solid squares in Figure 1. By contrast, in the complete design, both the top and bottom halves of composite faces can be the same or different (Figure 1). Another discrepancy is that in the standard design, participants were instructed to only make judgments of the top halves, whereas, in the complete design, cues are utilized to indicate whether participants should judge the top or the bottom halves for each trial. There is an ongoing debate on which of them is better for measuring holistic processing (Richler \& Gauthier, 2013, 2014; Richler et al., 2012; Rossion, 2013). While Richler and colleagues (2011) demonstrated that the composite effect measured by the standard design was vulnerable to response biases, Rossion (2013) argued that the effect assessed by the complete design consisted of extra attentional confounds. We adopted a modified complete design with judgments restricted to top halves of the composites; this is similar to the updated standard design (e.g., Busigny et al., 2014; Jacques \& Rossion, 2009, 2010; Laguesse \& Rossion, 2013; Retter \& Rossion, 2015; Rossion \& Retter, 2015), in which the "same congruent" (Figure 1) trial type is added to the standard design, reducing the response bias observed in the standard design (Rossion \& Retter, 2015). By not varying the target location, participants were only required to make identity judgments of the top half, which eliminated the potential attention confounds in the complete design (Rosenthal, Levakov, \& Avidan, 2018). In addition, with this version, both the complete design, i.e., the interaction between Congruency and Alignment, and the standard design, i.e., 
the differences between aligned and misaligned conditions (for same incongruent trials), can be calculated ${ }^{1}$.

On half the trials, irrelevant (bottom) facial halves were presented with CFS by using shutter glasses, in order to present them unconsciously. For the shutter glasses, when one lens opened, the other lens closed, and this oscillated at high frequency. Synchronized to this oscillation, the monitor also switched the stimuli displayed on the screen. Consequently, different stimuli were presented to different eyes with the shutter glasses. We displayed the continuous flashing stimuli to participants' dominant eyes and the experimental stimuli (i.e., faces) to their non-dominant eyes. As a result, participants should perceive the experimental stimuli unconsciously.

In Experiment 1a and 1b, we explored whether irrelevant facial halves influenced the processing of target facial parts, as is expected in the face composite effect, when the irrelevant halves were masked by CFS. In the CFS condition, composite faces were presented to the non-dominant eyes and the CFS, which covered the irrelevant (i.e., bottom) halves of both the study and test faces, was presented to the dominant eyes. Thus, participants should perceive the target halves (unmasked) consciously and the irrelevant halves (masked) unconsciously. In the monocular condition, a blank (i.e., gray) screen instead of the CFS was presented to the dominant eyes, and composite faces were still presented to the non-dominant eyes. Therefore, participants should perceive the whole composite face consciously. In

\footnotetext{
${ }^{1}$ Both the composite effect for the complete design (i.e., the interaction between Congruency and Alignment) and the composite effect for the standard design (i.e., the differences of accuracy for "same incongruent" trials between aligned and misaligned composites) are calculated in this study. For simplicity, in the text we will note the results of the complete design only, with additional information in a footnote if the standard design produced a different result.
} 
addition, to test whether the identity information of irrelevant facial halves can be perceived during the composite face task, catch trials were introduced in Experiment $1 \mathrm{~b}$.

\section{Experiment 1a and $1 b$}

\subsection{Methods}

\subsubsection{Participants}

Twenty $(14$ females, age range: $18-34$ years old, Mage $=22.35)$ and thirty $(21$ females, age range: $18-34$ years old, $M_{\text {age }}=22.90$ ) healthy adults with normal or corrected-tonormal vision participated in Experiment $1 \mathrm{a}$ and $1 \mathrm{~b}$ respectively in exchange for course credits or supermarket vouchers and gave written consent before completing the tasks. An additional four participants were excluded from Experiment $1 \mathrm{~b}$ due to different reasons (for one, more than $30 \%$ of response times in the monocular condition were shorter than $200 \mathrm{~ms}$, and for the other three, their sensitivity $d$ ' was negative in at least one of the conditions). This study was approved by the University of Auckland Human Participants Ethics Committee (Reference Number 016078).

\subsubsection{Stimuli}

Forty Caucasian face photos (20 females) from Ge et al. (2009) were used in this experiment. All the photos were converted to grey scale and cropped into an ellipse $(200 \times$ 256 pixels), which removed the color and contour information, such as from the hair and ears. The luminance of all the grey images was matched using the SHINE toolbox (Willenbockel et al., 2010) in Matlab. Each elliptical image was cut into two halves horizontally through the middle of the face, which was around the tip of the nose; an example stimulus not used in the 

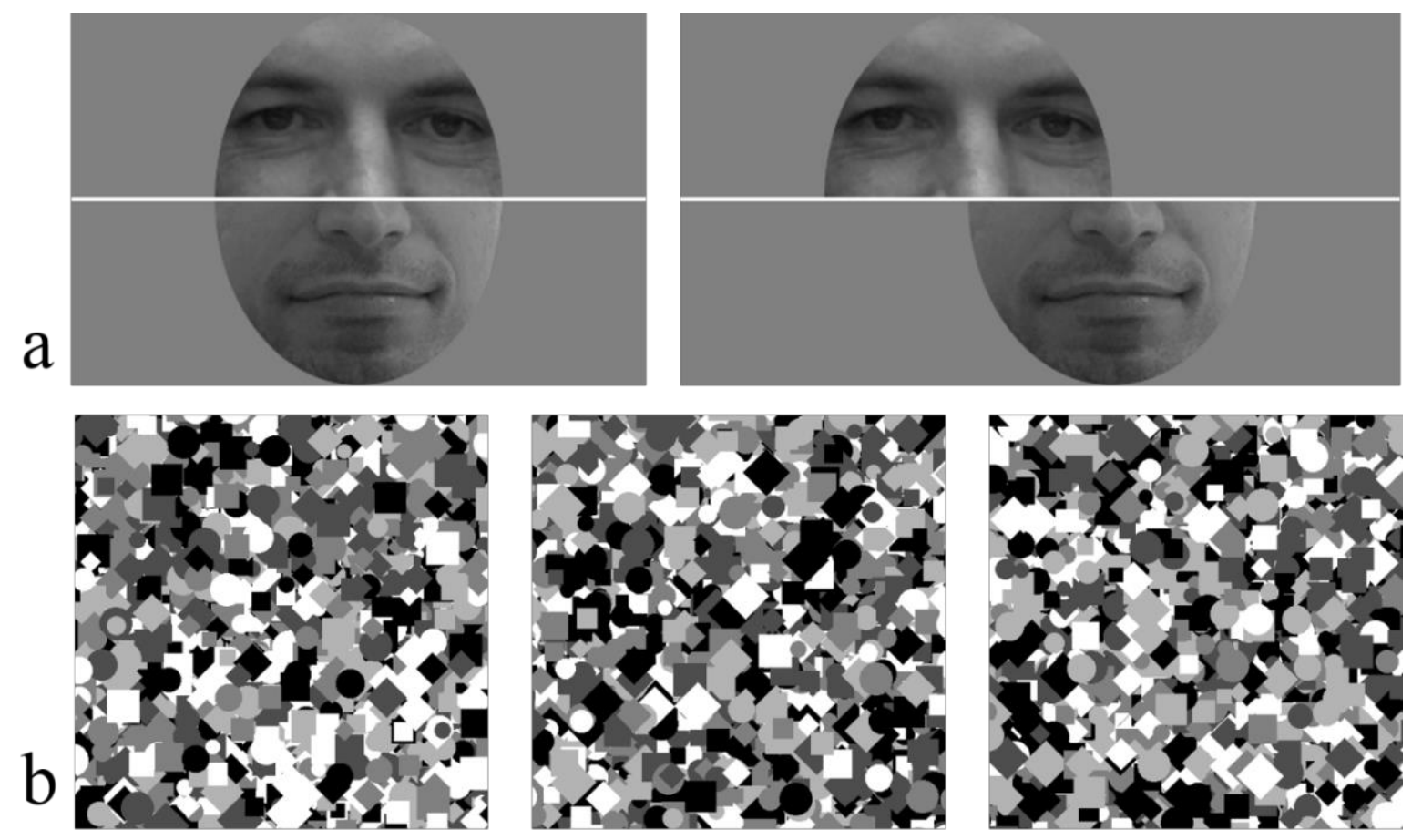

Figure 2. Example stimuli in Experiment 1. (a) Examples of the aligned and misaligned composite faces. The top and bottom facial halves were always from different identities. Aligned composites were created by aligning the two parts and misaligned composites were created by offsetting the bottom parts to the right by half of the facial width. Both exemplars were not used in the experiments. (b) Examples of Mondrian stimuli used in the experiment. They were made of varied sizes, numbers and grey-scale colors of shapes, including squares, circulars, and diamonds, randomly being located on a canvas.

experiment is shown in Figure 2a. Aligned composite faces were created by combining the top half of one identity and the bottom half of another identity. A 3-pixel-width white line was also utilized between the two halves to facilitate participants knowing clearly which parts of the faces were top and bottom halves (Rossion \& Retter, 2015). Therefore, the size of the aligned composite faces were $200 \times 259$ pixels $\left(5.68^{\circ} \times 7.36^{\circ}\right)$. Misaligned composite faces were created by offsetting the bottom half of the composite 100 pixels to the right, resulting in the size of misaligned faces being $300 \times 259$ pixels $\left(8.52^{\circ} \times 7.36^{\circ}\right)$. Twenty images of each gender were divided into five sets (four images in each set). Only the top and bottom halves of different identities from the same set were used to create the composite face images.

Stimuli used in the catch trials of Experiment $1 \mathrm{~b}$ were randomly chosen from the composite face stimuli for every participant. 
One hundred grey scale Mondrian masks were created by using Matlab scripts which were modified from Stein, Hebart, \& Sterzer (2011). Every Mondrian mask was made of varied sizes, numbers and grey-scale colors of shapes, including squares, circulars, and diamonds, randomly being located on a $500 \times 500$ pixels $\left(14.2^{\circ} \times 14.2^{\circ}\right)$ canvas. Examples of Mondrian masks are shown as Figure $2 b$.

\subsubsection{Apparatus}

This experiment was administrated using Matlab (Version 9.2, MathWorks, Natick, MA) with Psychtoolbox (Brainard, 1997). Stimuli were displayed on an ASUS VG278H monitor in $1920 \times 1080$ resolution with a refresh rate of $120 \mathrm{~Hz}$. Shutter glasses were utilized to present different images to participants' different eyes. Throughout the whole experiment, participants were seated on a comfortable chair in a darkened room with their chin on a chin rest, which was $60 \mathrm{~cm}$ away from the screen, giving responses via the keyboard.

\subsubsection{Procedures}

\subsubsection{Procedure of Experiment 1a}

After filling out the questionnaire about their demographic information, participants had their dominant eyes identified in the Miles test (Miles, 1930). Every participant had two tasks to complete, a continuous flash suppression (CFS) composite face task and a monocular composite face task. The order of completing the tasks was counterbalanced across participants.

In the composite face task with CFS (Figure 3a), each trial began with a fixation for $300 \mathrm{~ms}$, followed by a blank screen for another $200 \mathrm{~ms}$. Next, a study composite face was displayed to participants' non-dominant eyes while dynamic Mondrian masks that changed every $100 \mathrm{~ms}(10 \mathrm{~Hz})$ and covered the bottom half of the face were displayed to their dominant 
a
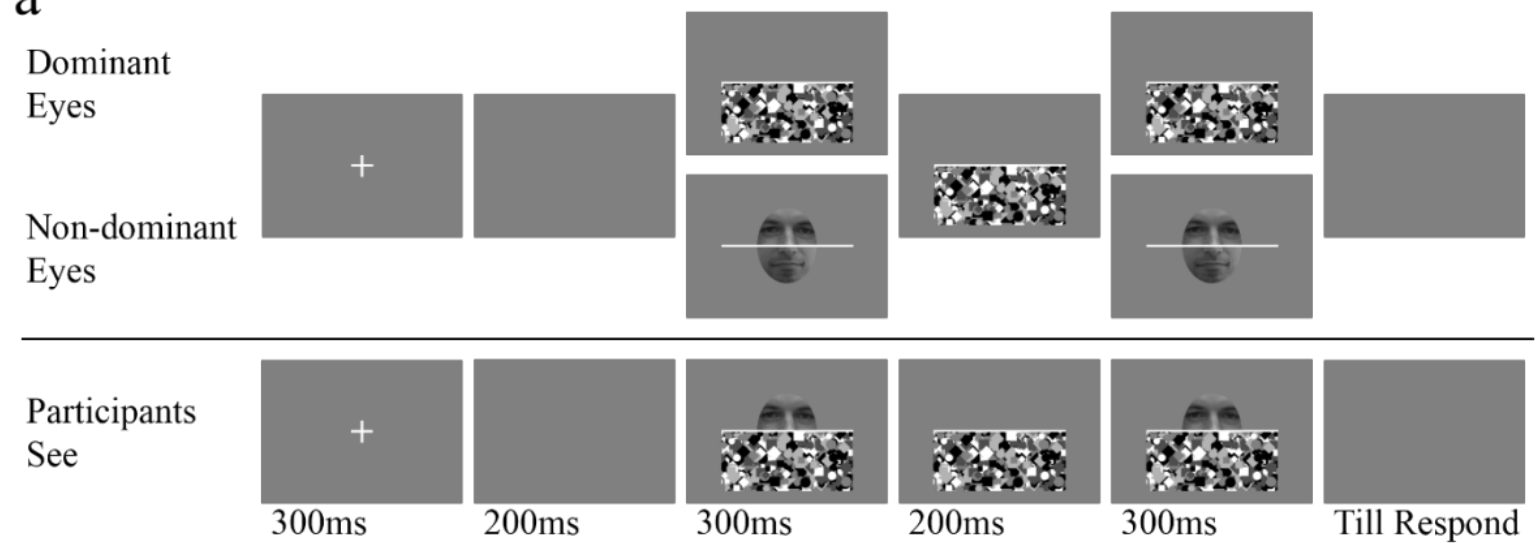

b

Dominant

Eyes

Non-dominant Eyes

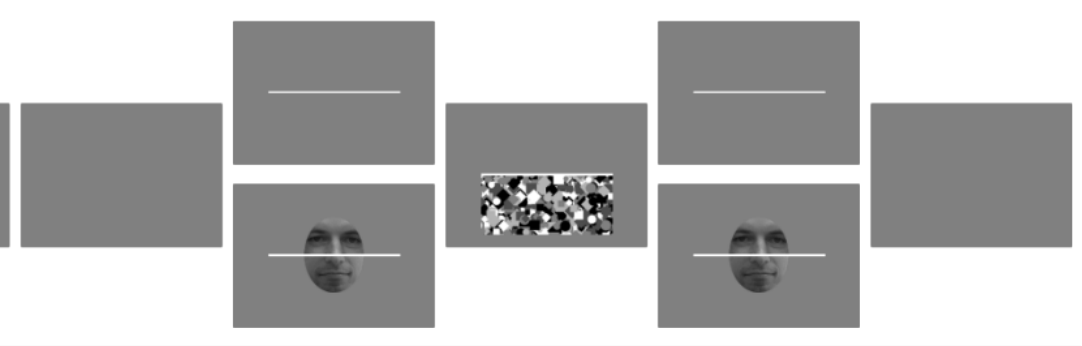

Participants

See
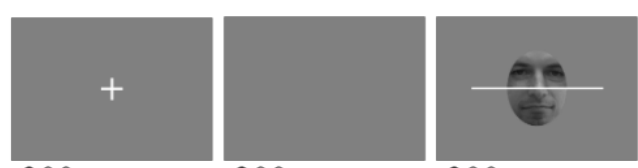

$300 \mathrm{~ms}$
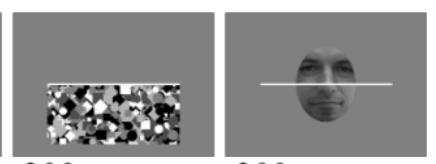

$300 \mathrm{~ms}$

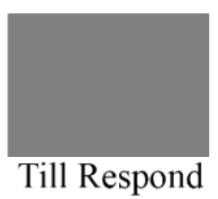

Figure 3. Procedures of composite tasks in Experiment 1. Participants were instructed to judge whether the top parts of the two faces were the same. (a) The procedure used in the CFS condition. The composite faces were always presented to participants' non-dominant eye and the Mondrian masking was presented to the dominant eye. Critically, the bottom halves of both study and test faces were covered by Mondrian masking. (b) The procedure used in the monocular condition. Its differences from the CFS procedure were that only the white line, instead of Mondrian masking, was presented to participants' dominant eyes when the two composite faces were displayed to the non-dominant eye.

eyes. After that, similar Mondrian masks continued to be presented on the screen but for both dominant and non-dominant eyes for $200 \mathrm{~ms}$. Finally, a test composite face was displayed to participants' non-dominant eyes, and the dynamic Mondrian masks stayed on the screen for their dominant eyes. The duration for study and test faces was $300 \mathrm{~ms}$. Following the test face, a response screen appeared and then disappeared as soon as one of the response keys was pressed. The interval between trials was 500ms. Response times (RT) were recorded from the onset of the test composite face to the time point that the response key was pressed. However, responses were not allowed during the first $300 \mathrm{~ms}$ (i.e., the duration of the test composite 
face). Only response times within three standard deviations of each condition were included in the following analysis.

Each trial in the monocular condition of the composite face task (Figure 3b) was similar to that in the task with CFS. The differences were that for the study and test faces in the monocular viewing condition, only the white line, but not the Mondrian masks, was displayed to participants' dominant eyes.

A 2 (Viewing condition: CFS vs. monocular; by block) $\times 2$ (Congruency: congruent vs. incongruent $) \times 2($ Alignment: aligned vs. misaligned $) \times 2$ (Identity: same vs. different $)$ within-subject design was utilized in Experiment 1a. There were 40 trials in each condition and, therefore, 640 trials in total.

\subsubsection{Procedure of Experiment 1b}

Similar to Experiment 1a, every participant in Experiment 1b completed a CFS block of trials and a monocular block of trials, but here these blocks included catch-trials $(6.25 \%$ of total trials) along with experimental trials. In addition, participants also completed a catchtrial only block. Catch trials were included to evaluate whether participants had conscious access to the irrelevant bottom-half components that typically induce the face composite effect. We were unable to evaluate these on experimental trials since requiring an explicit judgment of the bottom halves would be expected to affect the way participants performed the main identity judgment about top-halves.

The order of the two composite tasks were counterbalanced, after which participants completed the catch-only task.

The procedures of the CFS and monocular composite face tasks were similar to those in Experiment 1a, except that (1) participants were allowed to respond within the first $300 \mathrm{~ms}$ 


\section{a}

Dominant

Eyes

Non-dominant +

Eyes

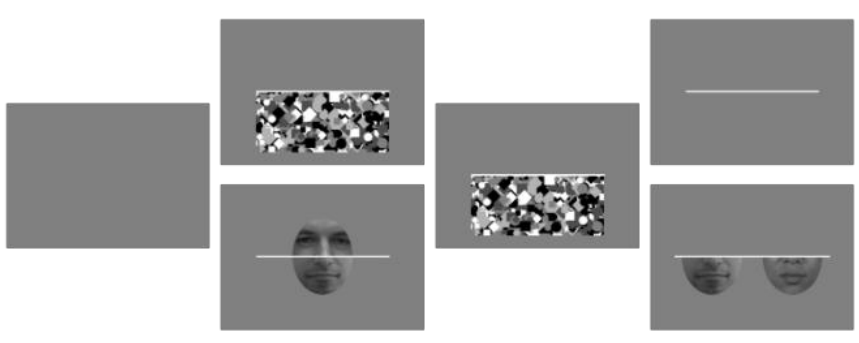

Participants

See
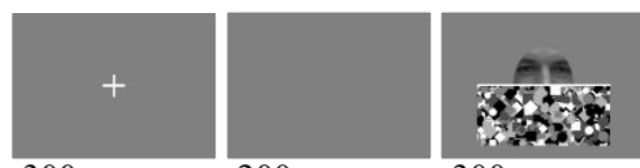

$300 \mathrm{~ms}$

$200 \mathrm{~ms}$

$300 \mathrm{~ms}$

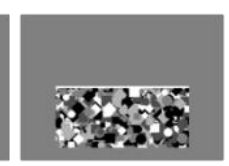

$200 \mathrm{~ms}$

Till Respond

$\mathrm{b}$

Dominant

Eyes

Non-dominant

Eyes
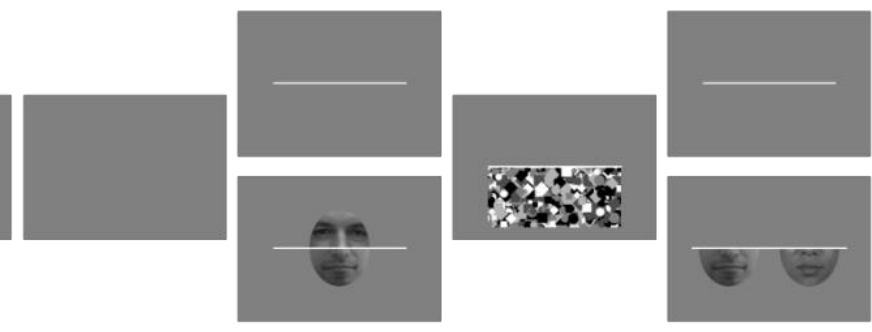

Participants

See

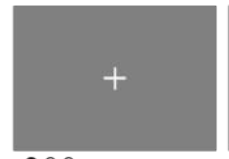

$300 \mathrm{~ms}$

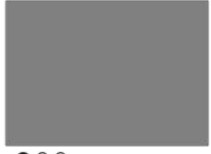

$200 \mathrm{~ms}$

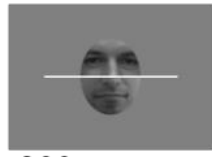

$300 \mathrm{~ms}$

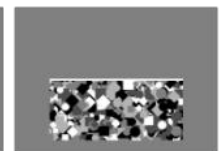

$200 \mathrm{~ms}$

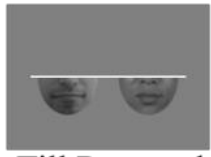

Till Respond

Figure 4. Procedures of catch trials in Experiment 1b. In the (a) CFS and (b) monocular conditions, the fixations, blanks, study faces and masks in catch trials were the same as those in the composite trials. Instead of showing the test composite faces, response screens were displayed in the catch trials. Participants were instructed to indicate which of the two bottom facial halves they saw for the study composite face. Only $6.25 \%$ of total trials were catch trials. The catch trials in the catch-only condition were the same as those in the (b) monocular condition but participants were instructed to only make judgment of the bottom halves.

during which the test faces were presented, and (2) catch trials were included in the composite face tasks.

The procedure for catch trials (Figure ) was the same as for trials in the composite tasks until the presentation of the test stimulus. At this point, instead of presenting the test composite face, a response screen appeared with the target bottom half along with a foil bottom half and participants were asked to choose which of them was identical to that presented as part of the study composite. The response screen disappeared as soon as one of the response keys was pressed. It should be noted that participants were informed of these 
catch trials before they started the tasks and it was also emphasized that they were asked to concentrate on the top halves during the whole experiment even though the task for the catch trials was to judge the bottom halves. In addition, only $6.25 \%$ of trials were catch trials, so participants were unlikely to adopt a strategy of focusing on the bottom halves. The catch trials were randomly intermixed with the non-catch trials in the composite tasks.

In the catch-only task, all catch trials were completed in succession, in the absence of composite task trials. Similar to the monocular condition, all the stimuli were only presented to participants' non-dominant eyes. By instructing participants to concentrate on the bottom halves of the composite faces, this block allowed us to measure the baseline perceptual difficulty of making the catch trial judgments.

The catch trial stimuli came from the composite face tasks. Specifically, one aligned, its corresponding misaligned, and one foil bottom half were randomly selected from each of 10 stimulus sets (see Stimuli in Experiment 1) for each composite task. Therefore, there were ten aligned and ten misaligned composite faces, and 20 trials in total for the catch trials embedding in the CFS and monocular tasks separately.

Since the catch trial stimuli were randomly selected for each composite task, the catch trial stimuli in the CFS and monocular tasks were not necessarily the same. In order to gain a better measurement of the baseline, the catch-only task included all the catch trials used in the CFS and monocular tasks. Therefore, there were 40 catch trials in the catch-only task.

\subsubsection{Data analysis}

The raw data were tidied up with the tidyverse library (Wickham, 2017) in R 3.5.2 (R Core Team, 2018) and R Studio 1.1.463 (RStudio Team, 2019). Statistical analyses were conducted in jamovi 0.9.5 (The jamovi project, 2019), in which the afex package (Singmann, 
Bolker, Westfall, \& Aust, 2019) and emmeans package (Lenth, 2019) were used. The R codes used are available online: https://osf.io/pjq7z/.

Since the composite task was the same in Experiment 1a and 1b, these data were firstly analyzed together to increase the statistical power of detecting the composite effect, especially in the CFS condition. Also, to reveal the potential impacts of embedded catch trials on the composite effect, the results of Experiment $1 \mathrm{a}$ and $1 \mathrm{~b}$ are then reported individually. Catch trials results in Experiment $1 \mathrm{~b}$ are reported at the end.

For the composite tasks, analysis was performed on the sensitivity $d$ ' and correct response times. First, to inspect whether the composite effect was observed in the CFS and monocular conditions, a 2 (Congruency: congruent vs. incongruent) $\times 2$ (Alignment: aligned vs. misaligned) repeated-measures ANOVA was performed for the two conditions separately. If the interaction between Congruency and Alignment was significant, simple effect analyses were used to make sure this significant interaction stemmed from better performance for congruent relative to incongruent conditions for aligned faces but not for misaligned ones. Moreover, the differences of composite effects between CFS and monocular conditions were explored by the three-way interaction in a 2 (Viewing condition: CFS vs. monocular) $\times 2$ (Congruency: congruent vs. incongruent $) \times 2$ (Alignment: aligned vs. misaligned $)$ repeatedmeasures ANOVA.

For catch trials, one-sample $t$-tests were performed to inspect whether participants could recognize the bottom halves of the study faces. Since there was no reason to expect the accuracy being lower than the chance level (0.5), we used one-tailed, instead of two-tailed, one sample $t$-tests. 


\subsection{Results}

\subsubsection{Combining Experiment 1a and 1b}

\subsubsection{Sensitivity $d^{\prime}$}

A 2 (Congruency: congruent vs. incongruent) $\times 2$ (Alignment: aligned vs. misaligned) repeated-measures ANOVA was conducted on the combined d' of Experiments $1 \mathrm{a}$ and $1 \mathrm{~b}$ for the two viewing conditions separately (Figure a). In the CFS condition, the interaction between Congruency and Alignment did not reach significance $(F<.001)$. In the monocular condition, significance was found for the critical interaction, $F(1,49)=21.65, M S E=.15, p$ $<.001$, partial $\eta^{2}=.31$. Simple effects analysis ${ }^{2}$ revealed that the sensitivity $d$ ' for congruent trials $(M=2.84,95 \% C I[2.633 .06])$ was higher than that for incongruent trials $(M=2.22$, $95 \% C I[2.002 .43])$ in the aligned condition $(p<.001)$, and no significant differences were found in the misaligned condition ( $\mathrm{p}>.30$; congruent, $M=2.63,95 \% C I$ [2.42 2.85], incongruent, $M=2.51,95 \% C I[2.302 .73])$. These results showed that the composite effect of sensitivity $d$ ' was only observed in the monocular condition.

Moreover, a 2 (Viewing condition: CFS vs. monocular) $\times 2$ (Congruency: congruent vs. incongruent $) \times 2($ Alignment: aligned vs. misaligned $)$ repeated-measures ANOVA performed on $d$ ' revealed the significant three-way interaction, $F(1,49)=16.70, M S E=.10$, $p<.001$, partial $\eta^{2}=.25$. This result shows that the interaction between Congruency and

\footnotetext{
${ }^{2}$ For all the simple effect analyses of the interaction between Congruency and Alignment, Bonferroni correction for two tests was applied (the reported $p$ values were the double of the uncorrected $p$ values), as only the differences between congruent and incongruent trials would be tested for the aligned and misaligned conditions separately.
} 

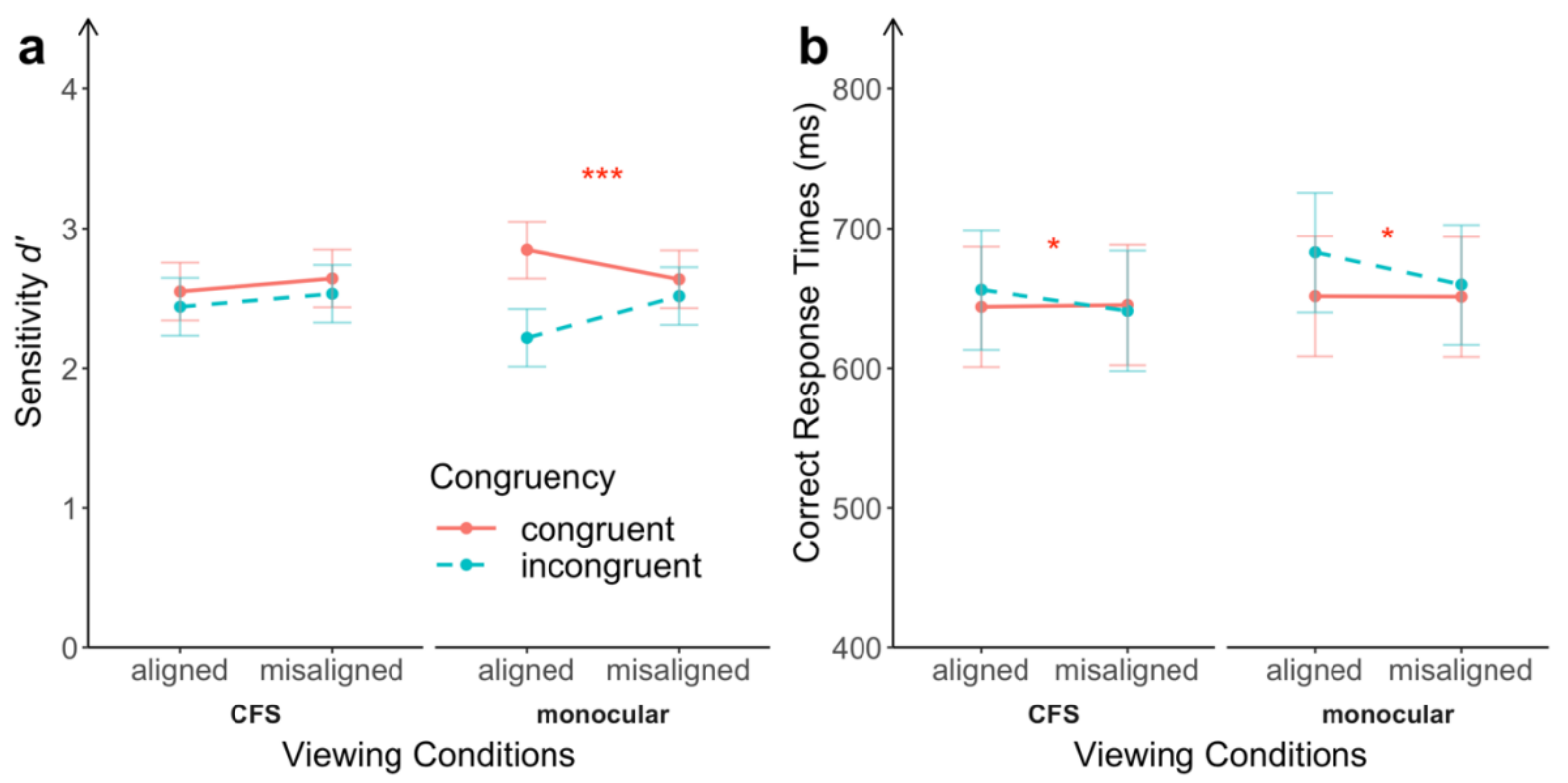

Figure 5. (a) Sensitivity $d$ ' and (b) correct response times as a function of Congruency and Alignment in the CFS and monocular viewing conditions for the combined data of Experiment 1a and 1b. Error bars represent $95 \%$ confidence intervals. Asterisks indicate significant interaction between Congruency and Alignment, i.e., the index of composite effects in the complete composite tasks $(*, p<.05 ; * * *, p<.001)$.

Alignment in the CFS condition was different from that in the monocular condition.

Specifically, the composite effect in the monocular condition was significantly larger than that in the CFS condition.

\subsubsection{Correct response times}

Similar 2 (Congruency: congruent vs. incongruent) $\times 2$ (Alignment: aligned vs.

misaligned) repeated-measures ANOVA was also conducted on the combined response times of Experiment $1 \mathrm{a}$ and $1 \mathrm{~b}$ for the two viewing conditions separately (Figure b). For the correct response times in the CFS condition, the critical interaction between Congruency and Alignment reached significance, $F(1,49)=5.10, M S E=659, p<.05$, partial $\eta^{2}=.09$. Simple effects analysis revealed that the correct response times for congruent trials $(M=644,95 \%$ $C I[601687])$ were marginally shorter than those for incongruent trials $(M=656,95 \% C I$ [613 699]) in the aligned condition $(p=.078)$, while there were no significant differences between congruent $(M=645,95 \% C I[602688])$ and incongruent trials $(M=641,95 \% C I$ [598684]) in the misaligned condition $(p>94)$. These results showed evidence that the 
composite effect was found for correct response times in the CFS condition, although it was rather weak. For the correct response times in the monocular condition, significant results were also observed for the interaction, $F(1,49)=4.65, M S E=1384, p<.05$, partial $\eta^{2}=.09$. Simple effects analysis displayed that the correct response times were shorter for the congruent trials $(M=651,95 \% C I[608695])$ compared with the incongruent trials $(M=683$, $95 \% C I[640726])$ in the aligned condition $(p<.001)$, and no significant difference was found in the misaligned condition (congruent: $M=651,95 \% C I$ [608 694]; incongruent: $M=$ $660,95 \% C I[617703] ; p>.46)$. These results show that the composite effect was observed in both CFS and monocular conditions.

Further, a 2 (Viewing condition: CFS vs. monocular) $\times 2$ (Congruency: congruent vs. incongruent $) \times 2$ (Alignment: aligned vs. misaligned) repeated-measures ANOVA was conducted on the correct response times to compare the composite effects observed in the CFS and monocular conditions. Results revealed that the three-way interaction did not reach significance $(F<.19)$, failing to provide the evidence that the composite effect in the CFS and monocular conditions were different from each other.

These results showed that the composite effect as measured by response times was found both in the CFS and monocular conditions.

\subsubsection{Separate analyses of the results of Experiment 1a and $1 \mathrm{~b}$}

Two (Congruency: congruent vs. incongruent) $\times 2$ (Alignment: aligned vs. misaligned) repeated-measures ANOVAs showed that in the CFS condition (Figure S1 and S2), the critical interaction between Congruency and Alignment did not reach significance for $d^{\prime}$ in Experiment $1 \mathrm{a}$ or $1 \mathrm{~b}(F<.07)$, or response times in Experiment $1 \mathrm{~b}, F(1,29)=2.09$, $M S E=926, p=.16$, partial $\eta^{2}=.07$. By contrast, the interaction was significant for response times in Experiment 1a, $F(1,19)=5.00, M S E=285, p<.05$, partial $\eta^{2}=.21$, which seems to 
display the composite effect in the CFS condition. However, the simple effects analyses showed that the differences between congruent $(M=542,95 \% C I[483,601])$ and incongruent trials $(M=548,95 \% C I[489,607])$ in the aligned condition were not significant $(p>.51)$ while the response times for congruent trials $(M=549,95 \% C I[490,608])$ were marginally longer than that for incongruent trials $(M=538,95 \% C I[479,596])$ in the misaligned condition $(p=.073)$. The pattern did not match that for the composite effect. Thus, this significant interaction cannot be taken as the evidence of the composite effect in the CFS condition.

In the monocular condition (Figure S1 and S2), the significant interaction between Congruency and Alignment was found for $d$ ' for both Experiment 1a, $F(1,19)=5.69$, MSE $=.16, p<.05$, partial $\eta^{2}=.23$, and $1 \mathrm{~b}, F(1,29)=16.28, M S E=.15, p<.001$, partial $\eta^{2}=.36$, but not for response times (Experiment 1a: $F(1,19)=1.64, M S E=646, p>.21$, partial $\eta^{2}$ $=.08$; Experiment 1b: $F(1,29)=3.12, M S E=1895, p=.088$, partial $\left.\eta^{2}=.10\right)$.

These results showed that when Experiment 1a and $1 \mathrm{~b}$ were analyzed separately, significant composite effects were only observed in the monocular condition for $d^{\prime}$ (see supplementary materials for detailed results).

\subsubsection{Catch trials in Experiment $1 b$}

One-tailed one-sample $t$ tests were conducted on accuracy of catch trials in each condition to explore whether the accuracy was above chance (Figure). The accuracy observed in the CFS and monocular conditions were not higher than chance (CFS aligned: $t(29)=0.94$, $p=.17$, Cohen's $d=.17$; CFS misaligned: $t(29)=-1.21, p=.88$, Cohen's $d=-.22$; monocular aligned: $t(29)=1.24, p=.11$, Cohen's $d=.23$; monocular misaligned: $t(29)=$ $2.11, p=.98$, Cohen's $d=-.39)$. However, accuracy in the catch-only conditions was significantly higher than chance (aligned: $t(29)=21.15, p<.001$, Cohen's $d=3.86$; 


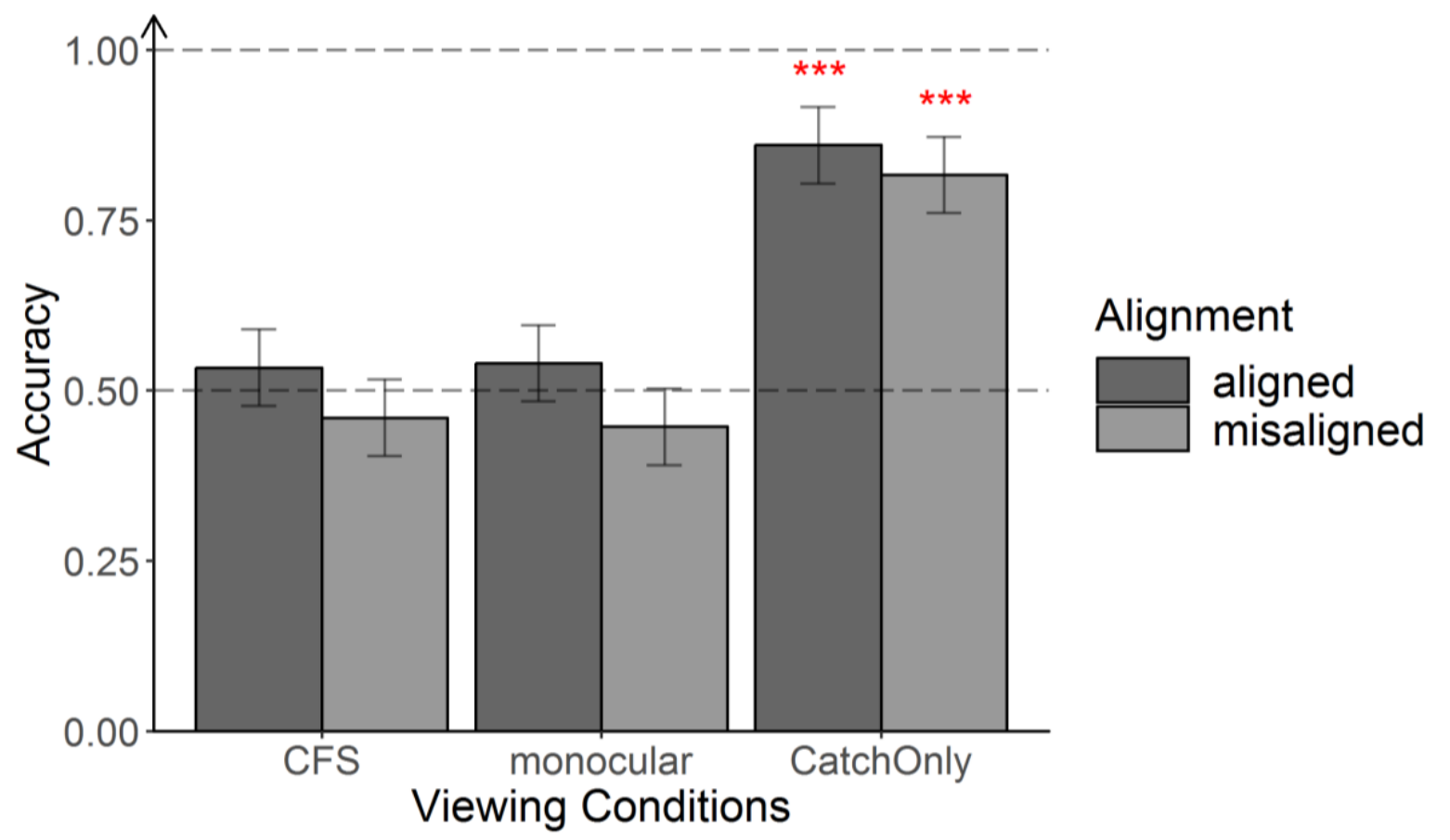

Figure 6. Accuracy as a function of Viewing condition and Alignment for catch trials in Experiment $1 \mathrm{~b}$. Error bars represent 95\% confidence intervals. Asterisks indicate that the performance was significantly above chance level $(50 \%)(* * *, p<.001)$.

misaligned: $t(29)=14.14, p<.001$, Cohen's $d=2.58)$. These results show that information about the bottom halves was perceptible, but only in the catch-trial condition. Surprisingly, judgments of bottom halves were at chance for the monocular condition as well as for the CFS condition, showing that when catch trials were embedded randomly within experimental trials, participants were unable to consciously report the identity of bottom halves, even when they were unmasked.

\subsection{Discussion}

Experiment 1 displays that the composite effect was found for response times in the CFS condition and both the sensitivity $d$ ' and response times in the monocular condition, at least when data of the two parts were analyzed together. Thus, when the bottom halves of study and test faces were masked, the speed in processing of top halves was influenced by the bottom halves, and when the bottom halves of study and test faces were unmasked, both the 
accuracy and speed in processing of top halves were influenced by the bottom halves. Furthermore, the composite face effect in the monocular condition was stronger than that in the CFS condition, indicating that the influence of bottom halves was reduced when they were masked.

The performance of catch trials in the CFS conditions was not significantly better than chance, showing no evidence that the bottom halves could be identified when they were masked. Surprisingly, the accuracies in the monocular conditions were not significantly higher than the chance level, either. This unexpected result showed that the bottom halves of study faces could not be identified better than expected by chance even when faces were not covered by CFS. Nevertheless, at the same time, the bottom half information did influence the processing of the top halves, as shown by the stronger composite effect in the monocular condition. The above evidence implies that the composite effect in the monocular condition likely reflects preconscious processing (Dehaene, Changeux, Naccache, Sackur, \& Sergent, 2006) rather than conscious processing; this will be addressed in more depth in the General Discussion. Performance on catch trials was significantly above chance in the catch-only condition, showing that participants were able to make judgments about identity information of the bottom halves where they were asked to focus only on that information.

In Experiment 1, the bottom facial halves of both study and test faces were covered by masking in the CFS condition. It is unclear whether the reduced composite effect with masking required both study and test images to be masked. Therefore, in Experiment 2, the study image was unmasked in both CFS and monocular conditions, whereas masking occurred on the test image in the CFS, but not monocular, condition. In addition, similar to Experiment $1 \mathrm{~b}$, catch trials were also embedded in composite tasks to scrutinize whether the bottom halves of the test face could be identified in the CFS and monocular conditions. 


\section{Experiment 2a and $2 b$}

\subsection{Methods}

\subsubsection{Participants}

Twenty (10 females, age range: $19-31$ years old, $\left.M_{\text {age }}=23.80\right)$ and thirty $(21$ females, age range: $18-34$ years old, $M_{\text {age }}=22.23$ ) healthy participants with the same criteria were recruited for Experiment $2 \mathrm{a}$ and $2 \mathrm{~b}$, respectively. An additional two participants were excluded from Experiment 2a because the response times of more than $30 \%$ trials in the monocular condition were shorter than $200 \mathrm{~ms}$ or his/her sensitivity $d$ ' was negative for all the monocular conditions. Another participant was excluded from Experiment $2 \mathrm{~b}$ due to sensitivity $d$ ' being negative in two conditions.

\subsubsection{Stimuli, apparatus, and data analysis}

The stimuli, apparatus, and data analysis procedures were the same as Experiment 1.

\subsubsection{Procedures}

\subsubsection{Procedure of Experiment 2a}

The procedures of the composite tasks were similar to those in Experiment 1a. One of the differences was that in this experiment, only the test, but not the study, faces were covered by the Mondrian masks in the CFS condition (Figure). The other difference was that responses were allowed after the onset of the test composite faces, which was the same as Experiment $1 b$. 
a
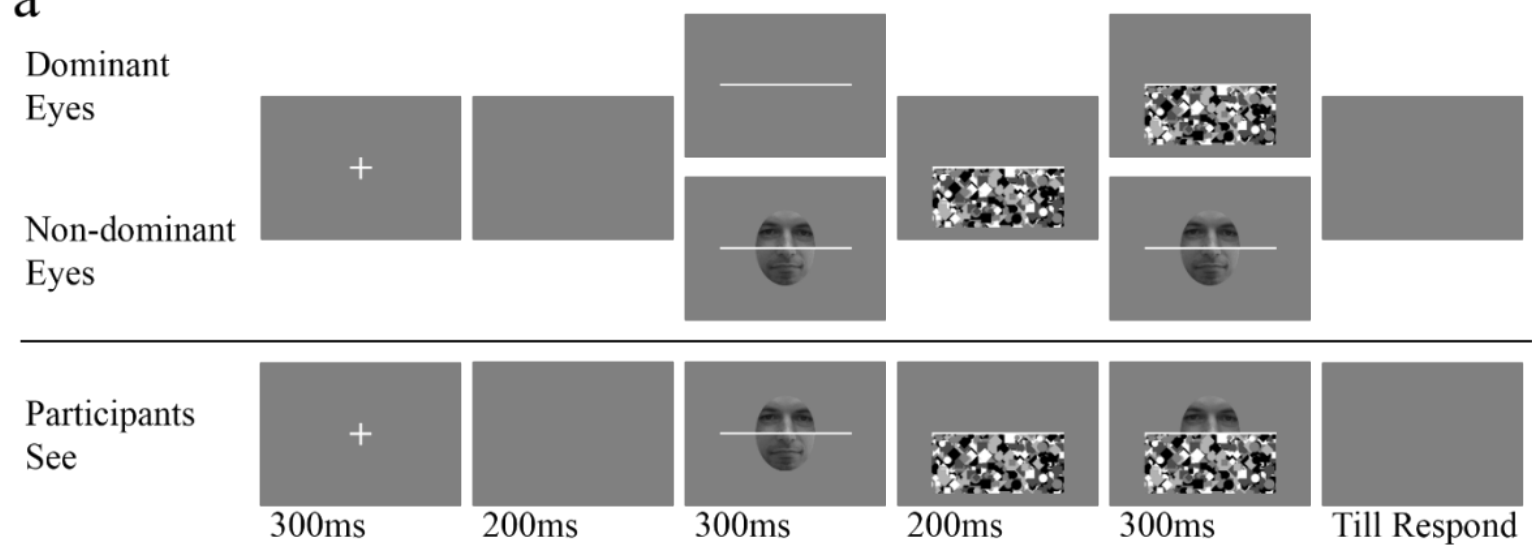

$\mathrm{b}$

Dominant

Eyes

Non-dominant

Eyes

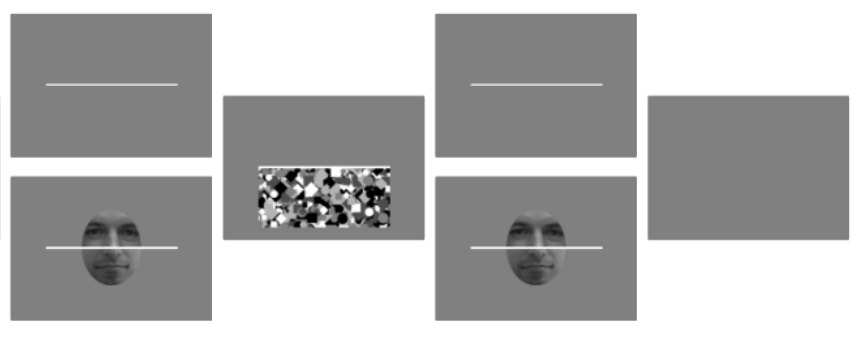

Participants

See
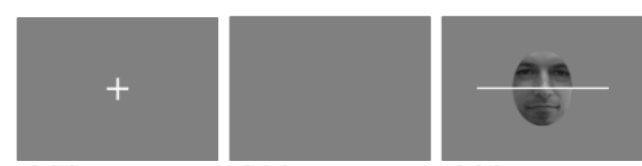

$300 \mathrm{~ms}$
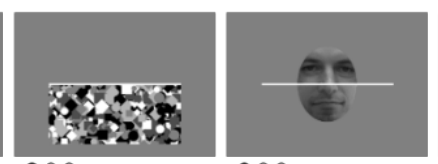

$300 \mathrm{~ms}$

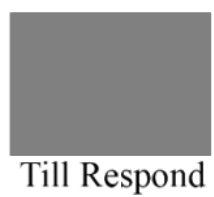

Figure 7. Procedures of composite tasks in Experiment 2a. Participants were instructed to judge whether the top parts of the two faces were the same. (a) The procedure used in the CFS condition. The composite faces were always presented to participants' non-dominant eye and the Mondrian masking was presented to the dominant eye. Critically, only the bottom halves of test, but not the study, faces were covered by Mondrian masking. (b) The procedure used in the monocular condition, which was the same as Experiment 1. Its only differences from the CFS procedure were that only the white line, instead of Mondrian masking, was presented to participants' dominant eye when the test face was displayed to the non-dominant eye.

\subsubsection{Procedure of Experiment 2b}

The procedures of the CFS and monocular composite face tasks were similar to those in Experiment 2a. The only difference was that catch trials were interleaved with composite trials in this experiment.

The design (Figure) of the catch trials were similar to those in Experiment 1b. One of the differences was that in Experiment $1 \mathrm{~b}$, catch trials examined if the bottom halves of study composite faces could be identified, while in Experiment 2b, it was the bottom halves of test composite faces that were inspected in the catch trials. Another difference was that in this 


\section{$\mathrm{a}$}

Dominant

Eyes

Non-dominant

Eyes
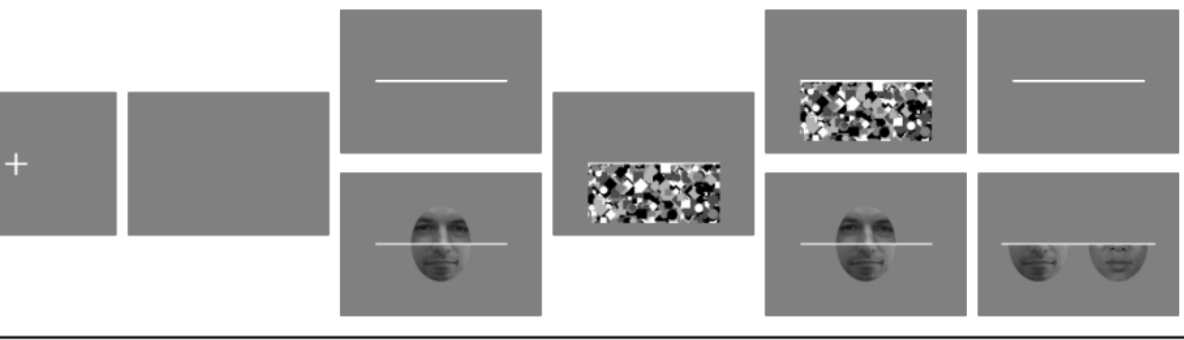

Eyes
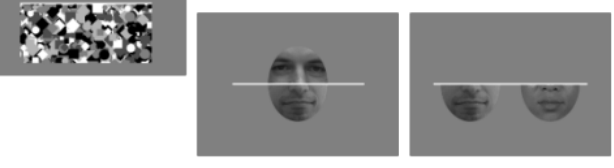

Participants
Perceive
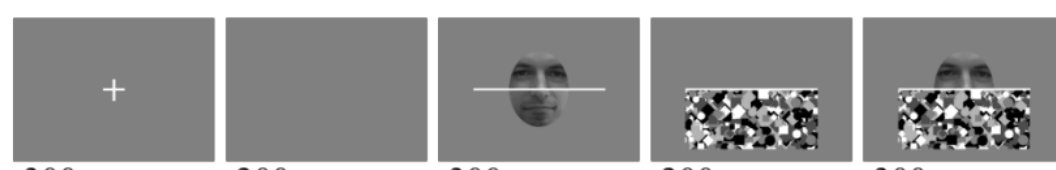

$300 \mathrm{~ms}$

$200 \mathrm{~ms}$

$300 \mathrm{~ms}$

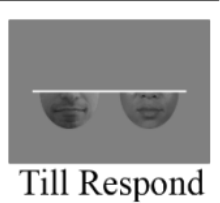

b

Dominant
Eyes

Non-dominant

Eyes
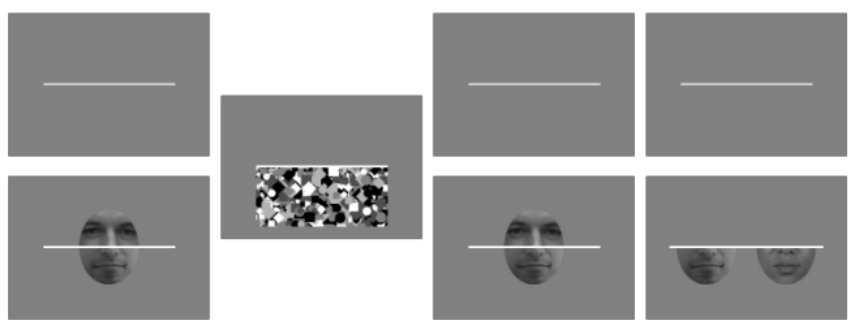

Participants

Perceive
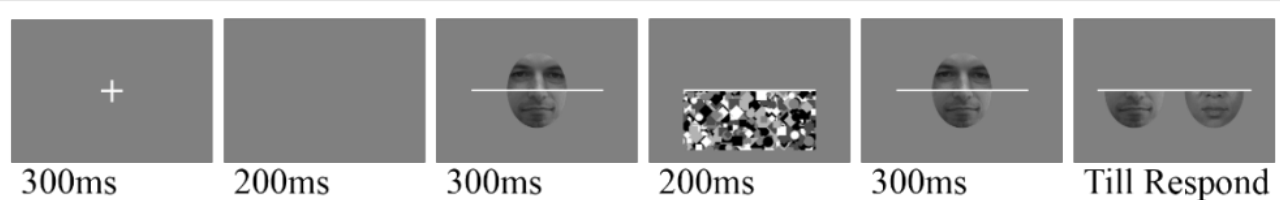

Figure 8. Procedures of catch trials in Experiment 2b. In the (a) CFS and (b) monocular conditions, the fixations, blanks, study faces, masking and test composite faces in catch trials were the same as those in the composite trials. Instead of showing the response screen for the composite task, response screens for catch trials were displayed. The response keys for composite tasks and catch trials were different. The catch trials in the catch-only condition were the same as those in the (b) monocular condition but participants were instructed to only make judgment of the bottom halves.

experiment, the response keys used for catch trials were different from those for composite tasks, as using the same response keys for both tasks might create confusion regarding the responses for composite tasks with those of catch trials. Moreover, in Experiment 2b, there were 10 catch trials in total for each composite task and 20 trials for the catch-only task.

Like Experiment 1b, catch trials were also tested separately without composite trials in the catch-only task. Participants were instructed to focus on bottom halves of the second (i.e., test) composite faces and completed all the trials in succession. This block allowed us to measure the baseline perceptual difficulty of making identity judgments for bottom halves of test faces. 


\subsection{Results}

\subsubsection{Combining Experiment 2a and $2 b$}

\subsubsection{Sensitivity $d^{\prime}$}

Two (Congruency: congruent vs. incongruent) $\times 2$ (Alignment: aligned vs misaligned) repeated-measures ANOVAs were conducted on the sensitivity $d$ ' of Experiments $2 \mathrm{a}$ and $2 \mathrm{~b}$ for the two viewing conditions separately (Figurea). For the CFS condition, the critical interaction was marginally significant ${ }^{3}, F(1,49)=3.04, M S E=.20, p$ $=.087$, partial $\eta^{2}=.06$. Simple effects analysis showed that the sensitivity $d$ ' of congruent trials $(M=2.79,95 \% C I[2.573 .00])$ was better than for incongruent trials $(M=2.53,95 \%$ $\left.C I\left[\begin{array}{ll}2.31 & 2.74\end{array}\right]\right)$ in the aligned condition $(p<.01)$, while there were no significant differences between congruent $(M=2.75,95 \% C I[2.542 .97])$ and incongruent trials $(M=2.72,95 \% C I$ [2.50 2.93]) in the misaligned condition $(p>.99)$. These results indicate that the composite effect was observed in the CFS condition for sensitivity $d$, , but with weak evidence. For the monocular condition, the interaction was found to be significant, $F(1,49)=32.7, M S E=.20$, $p<.001$, partial $\eta^{2}=.40$. Simple effects analysis displayed higher sensitivity $d$ ' for congruent trials $(M=2.97,95 \% C I[2.763 .17])$ than that for incongruent trials $(M=2.14$, $95 \% C I[1.942 .35])$ in the aligned condition $(p<.001)$, but the differences between congruent $(M=2.78,95 \% C I[2.572 .98])$ and incongruent $(M=2.68,95 \% C I[2.472 .88])$ trials in the misaligned condition were not significant $(p>.52)$.

\footnotetext{
${ }^{3}$ In the standard design analyses, accuracy of aligned trials were worse than those of misaligned trials in the CFS condition $(t(84.27)=-2.44, p<.05)$, showing the composite effect in the CFS condition.
} 

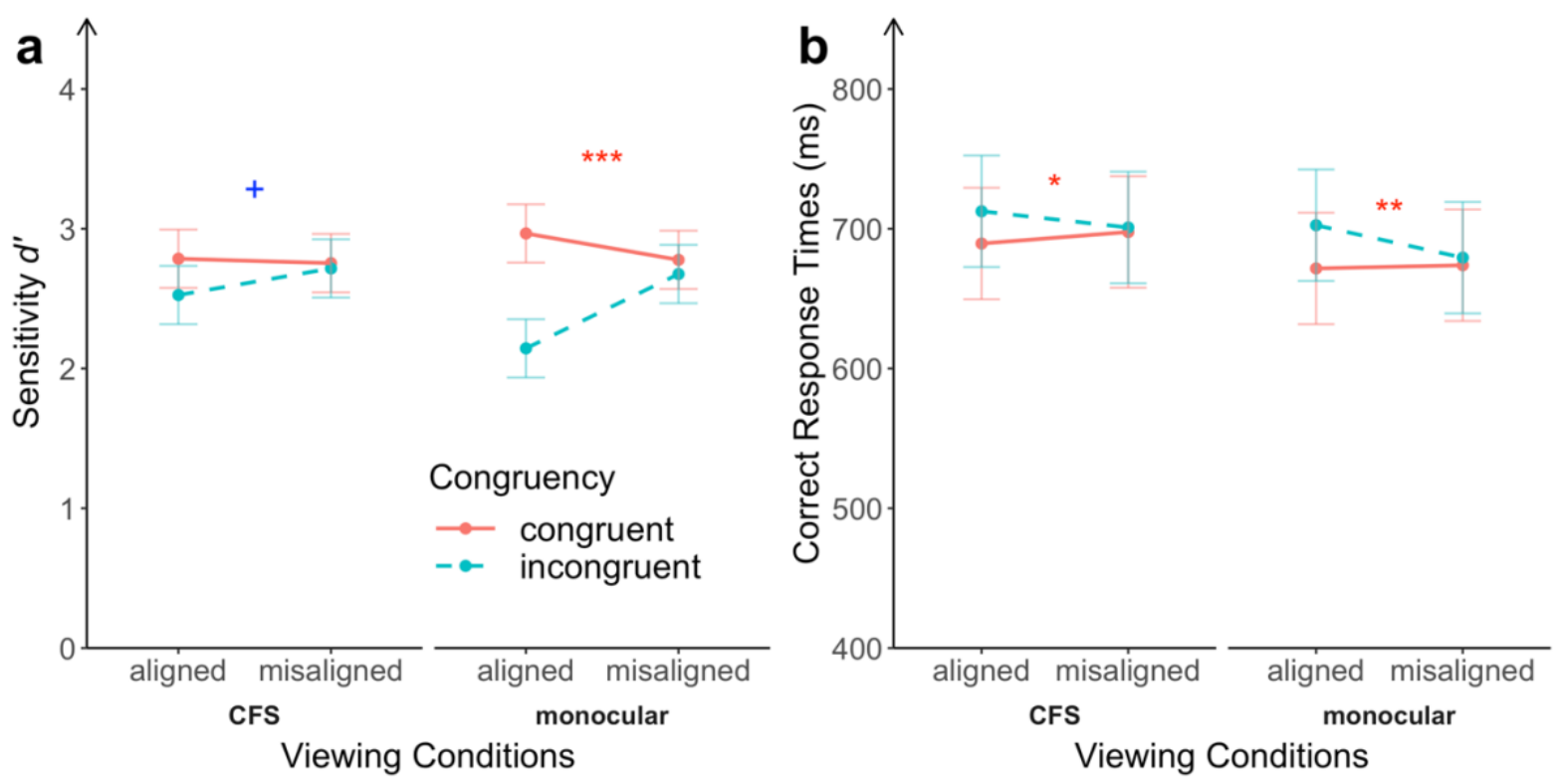

Figure 9. (a) Sensitivity $d^{\prime}$ ' and (b) correct response times as a function of Congruency and Alignment in the CFS and monocular viewing conditions for the combined data of Experiment $2 \mathrm{a}$ and $2 \mathrm{~b}$. Error bars represent $95 \%$ confidence intervals. Asterisks and plus signs indicate (marginal) significant interaction between Congruency and Alignment, i.e., the index of composite effects in the complete composite tasks $(+, p=.087 ; *, p<.05 ; * *, p<.01 ; * * *, p<.001)$.

A 2 (Viewing condition: CFS vs. monocular) $\times 2$ (Congruency: congruent vs. incongruent) $\times 2$ (Alignment: aligned vs. misaligned) repeated-measures ANOVA was used to inspect the differences between the composite effects in the CFS and monocular conditions. The three-way interaction was found to be significant, $F(1,49)=9.71, M S E$ $=.16, p<.01$, partial $\eta^{2}=.17$, suggesting the composite effect in the CFS condition was distinctive from that in the monocular condition. Specifically, the composite effect in the monocular condition was significantly larger than that in the CFS condition.

These results show that the composite effect in sensitivity $d$ ' was observed in both the CFS and monocular conditions. Furthermore, the composite effect in the monocular condition was larger than that in the CFS condition, consistent with the results of Experiment 1.

\subsubsection{Correct response times}

Repeated-measures ANOVAs of Congruency and Alignment were conducted for the two conditions separately on response times (Figureb). Similar to the results for the combined 
data of Experiment 1a and 1b, significant interactions between Congruency and Alignment were found for both the CFS, $F(1,49)=4.21, M S E=1169, p<.05$, partial $\eta^{2}=.08$, and monocular, $F(1,49)=8.01, M S E=1033, p<.01$, partial $\eta^{2}=.14$, conditions. Simple effects analysis in the CFS condition showed that the correct response times for congruent trials ( $M$ $=689,95 \% C I[648731])$ were shorter compared with those for incongruent trials $(M=713$, $95 \% C I[671754])$ in the aligned condition $(p<.001)$, while no significant differences between them were found in the misaligned condition ( $p>.99$; congruent: $M=698,95 \% C I$ [656 739]; incongruent: $M=701,95 \% C I$ [659 743]). Simple effects analysis in the monocular condition revealed that the correct response times for congruent trials $(M=672$, $95 \% C I[633710])$ were shorter than those for incongruent trials $(M=703,95 \% C I[664$ $741])$ in the aligned condition $(p<.001)$, and, however, no differences were found between the congruent $(M=674,95 \% C I[635712])$ and incongruent $(M=679,95 \% C I[641718])$ trials in the misaligned condition $(p>.83)$. These findings indicate that the composite effect for correct response times was found in the both the CFS and the monocular condition.

Moreover, a 2 (Viewing condition: CFS vs. monocular) $\times 2$ (Congruency: congruent vs. incongruent $) \times 2($ Alignment: aligned vs. misaligned $)$ repeated-measures ANOVA on correct response times showed the three-way interaction did not reach significance $(F<.17)$, which did not provide evidence for significant differences between the composite effect in the CFS and monocular conditions for correct response times.

The above results showed that the composite effect of correct response times was found in both the CFS and monocular conditions, and no significant differences between them were observed. 


\subsubsection{Separate analyses of Experiment 2a and 2b}

Similar 2 (Congruency: congruent vs. incongruent) $\times 2$ (Alignment: aligned vs. misaligned) repeated-measures ANOVAs showed that in the CFS condition (Figure S3 and S4), the interaction between Congruency and Alignment were found to be nonsignificant for $d^{\prime}$ of Experiment 2a, $F(1,19)=1.01, M S E=.17, p>.32$, partial $\eta^{2}=.05$, Experiment $2 \mathrm{~b}$, $F(1,29)=1.98, M S E=.23, p>.17$, partial $\eta^{2}=.06$, or response times of Experiment $2 \mathrm{~b}, F$ $<.46$. By contrast, this critical interaction reached marginal significance ${ }^{4}$ for response times in Experiment $2 \mathrm{a}, F(1,19)=4.16, M S E=1813, p=.056$, partial $\eta^{2}=.18$.

In the monocular condition (Figure S3 and S4), the significant interaction between Congruency and Alignment was found for $d$ ' of Experiment 2a, $F(1,19)=4.83, M S E=.29, p$ $<.05$, partial $\eta^{2}=.20$, Experiment $2 b, F(1,29)=40.38, M S E=.13, p<.001$, partial $\eta^{2}=.58$, and responses times of Experiment $2 b, F(1,29)=6.73, M S E=1895, p<.05$, partial $\eta^{2}=.19$, but not for response times of Experiment 1a, $F(1,19)=1.41, M S E=609, p=.25$, partial $\eta^{2}$ $=.07$.

${ }^{4}$ In the standard design analyses, response times of aligned trials were longer than those of misaligned trials in the CFS condition $(t(37.99)=2.87, p<.05)$, showing the composite effect in the CFS condition. 


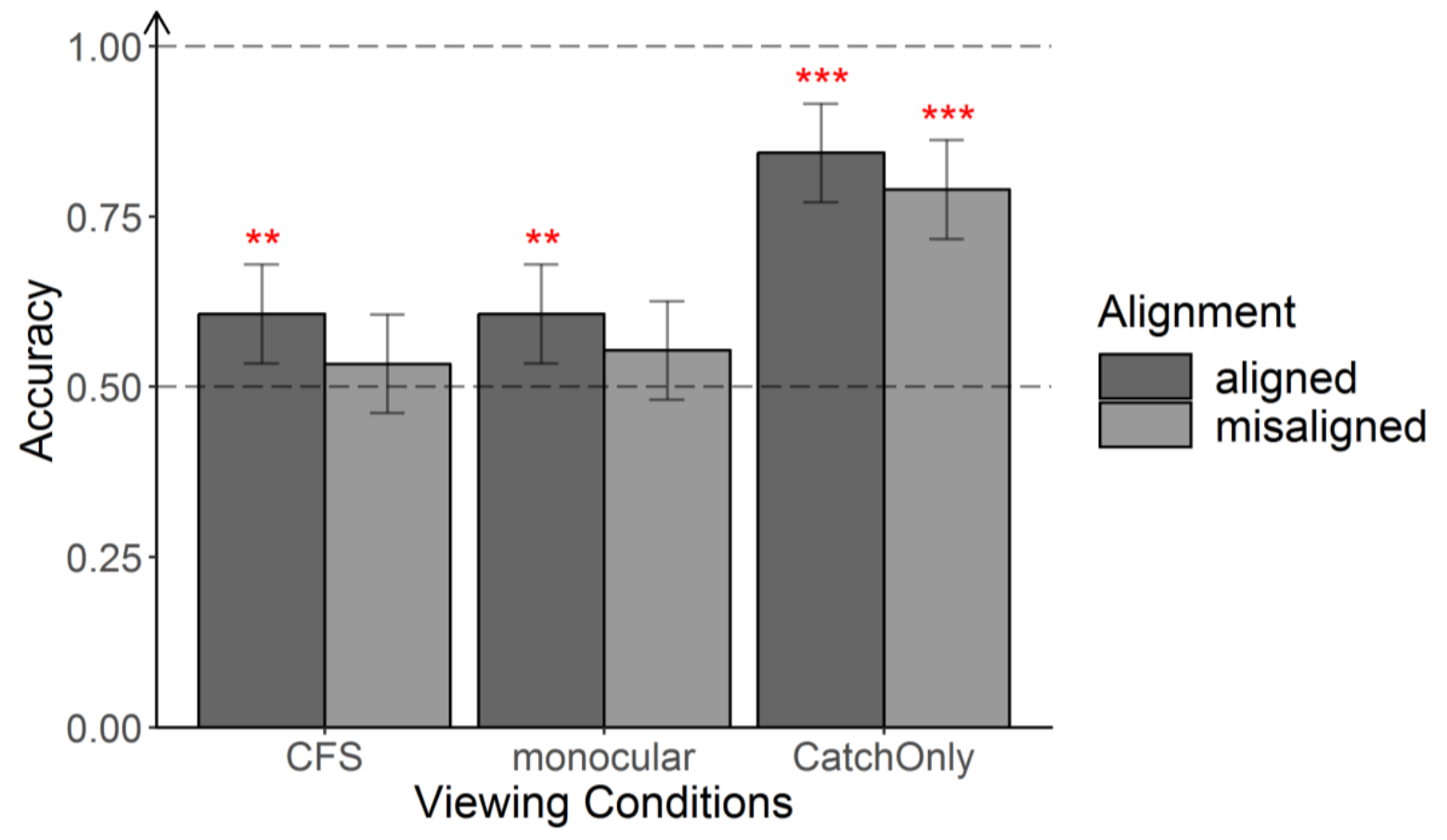

Figure 10. Accuracy as a function of Viewing condition and Alignment for catch trials in Experiment $2 \mathrm{~b}$. Error bars represent $95 \%$ confidence intervals. Asterisks indicate that performance was significantly above chance level $(50 \%)(* *, \mathbf{p}<.01, * * *, \mathbf{p}<.001)$.

These results showed that when Experiment 2a and $2 \mathrm{~b}$ were analyzed separately, significant composite effects were mainly observed in the monocular condition, but not in the CFS condition (see supplementary materials for detailed results).

\subsubsection{Catch trials in Experiment 2b}

Similar to Experiment 1b, one-tailed one-sample $t$-tests were conducted on the accuracy of catch trials in each condition to explore whether participants could recognize the bottom halves of the test faces (Figure). The accuracies in the aligned CFS and monocular conditions were significantly better than the chance level (CFS aligned: $t(29)=2.74, p<.01$, Cohen's $d=.50$; monocular aligned: $t(29)=2.66, p<.01$, Cohen's $d=.49$ ) while the accuracies in the misaligned conditions were not (CFS misaligned: $t(29)=.86, p=.20$, Cohen's $d=.16$; monocular misaligned: $t(29)=1.14, p=.13$, Cohen's $d=.21)$. The accuracies in the catch-only conditions were both higher than the chance level (aligned: $t(29)$ $=13.61, p<.001$, Cohen's $d=2.49$; misaligned: $t(29)=11.54, p<.001$, Cohen's $d=2.11)$. 


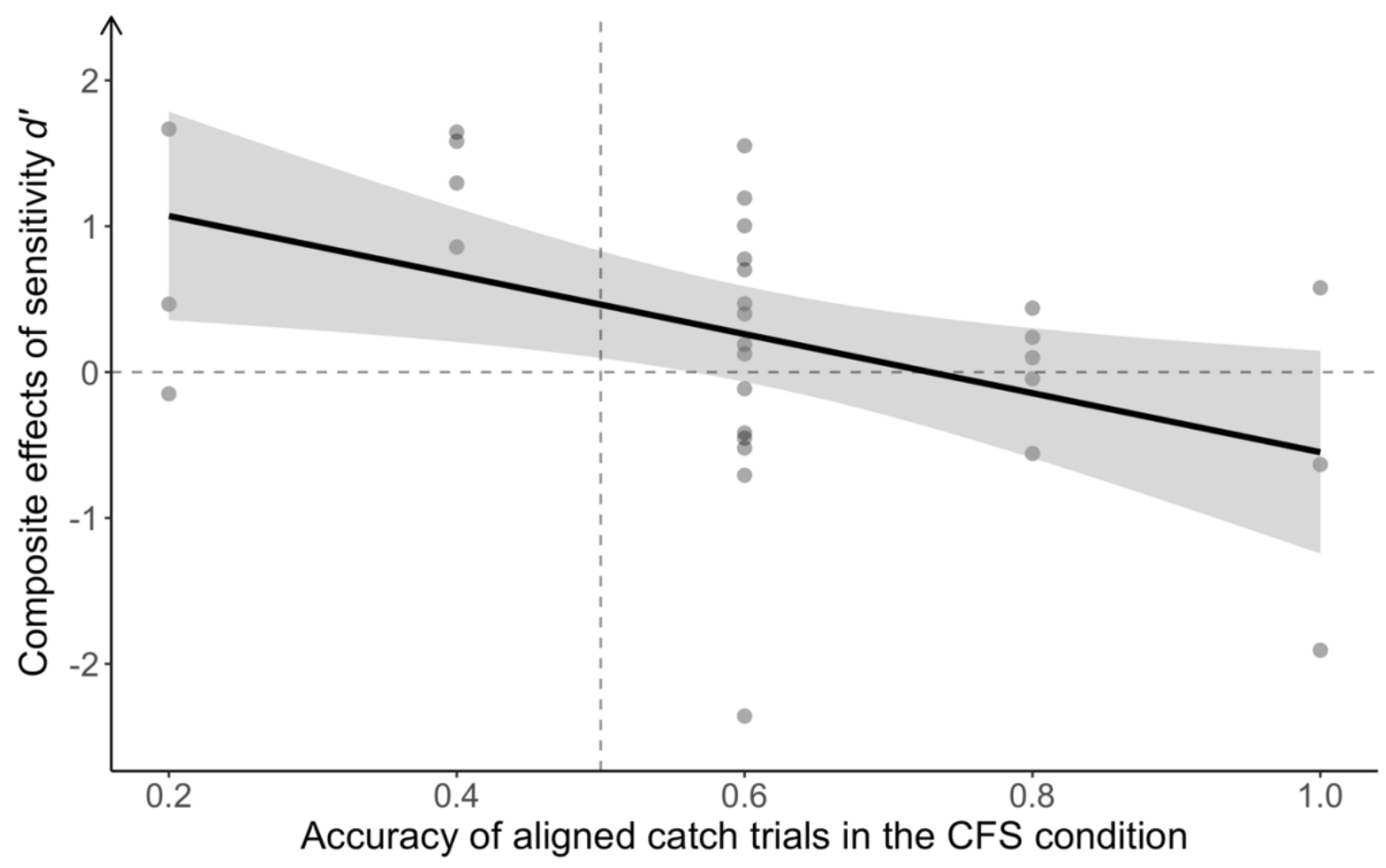

Figure 11. Relationships between the accuracy of aligned catch trials and the composite effect in the CFS condition. The black straight line denotes the regression line with accuracy as the covariate and the composite effects as the dependent variable. The shaded area denotes the $95 \%$ confidence intervals of the regression line. Participants with higher accuracy showed weaker composite effects. The vertical dashed line corresponds to participants who would perform at chance in catch trials. Importantly, the corresponding composite effects would be larger than 0 denoted by the horizontal dashed line.

These results suggest that participants have some access to the information of bottom halves of test faces in all the aligned conditions and the misaligned catch-only task.

The above-chance performance in the aligned CFS catch trials indicates that to some extent, participants could see the bottom facial halves covered by CFS, which might further result in the significant composite effects in the CFS condition. To explore whether participants who were more conscious of the bottom halves showed greater composite effects, we performed linear regressions with accuracy of catch trials for the CFS aligned condition as the covariate and the composite effect of $d^{\prime}$ ' (i.e., $\left[\left(d^{\prime}\right.\right.$ aligned/congruent $d^{\prime}$ aligned/incongruent $)$ - $\left(d^{\prime}\right.$ misaligned/congruent - $d^{\prime}$ misaligned/incongruent $\left.)\right]$; e.g., Chua, Richler, \& Gauthier, 2014; Wang et al., 2019) as the dependent variable. In particular, accuracy of catch trials being $50 \%$ was used as the Intercept, which tested whether a participant with chance performance in catch trials would show composite effects. Results (Figure 11) showed that 
participants with higher accuracy in catch trials showed weaker composite effects, $\beta=-2.02, t$ $=-2.66, p=.013, r(28)=-0.45$. This result indicates that if the conscious perception of bottom facial halves had any impact on the composite effect in the CFS condition, it would lead to smaller composite effects. In addition, a significant composite face effect would be observed for a participant with chance performance in catch trials, $\alpha($ Intercept $)=0.463, t=$ 2.58, $p=.015$. All of this evidence suggests that the significant composite effects observed in the CFS condition of Experiment $2 \mathrm{~b}$ were not likely because participants had some access to the identity information of the bottom halves.

\subsection{Discussion}

Similar to Experiment 1, the composite effect was found in both the CFS and monocular conditions, at least when Experiment $2 \mathrm{a}$ and $2 \mathrm{~b}$ were analyzed together. In addition, a stronger composite effect was still found in the monocular condition relative to the CFS condition. These findings suggest that no matter whether the bottom halves of test faces were masked or unmasked, the processing of the top halves was influenced by the bottom halves. However, the influence when the bottom halves of test faces were masked was smaller than when they were unmasked.

Also similar to Experiment 1, when experiments were analyzed separately, a significant composite effect was only observed in the monocular condition. This discrepancy might be due, again, to the low power of not having enough sample size when the experiments were analyzed respectively.

Consistent with the results of catch trials in the catch-only condition of Experiment $1 \mathrm{~b}$, the performance of catch trials in the catch-only condition was much better than that expected by chance, suggesting participants were able to make judgments about identity 
information in the bottom halves of test faces. For the catch trials embedded in the CFS or monocular composite face tasks, participants were only able to identify the bottom halves of aligned test faces, and even then only at slightly better than chance levels. These results indicate that only the bottom halves of aligned faces were identifiable, no matter if they were presented in the CFS or monocular conditions. Additional analyses showed that the stronger composite effects in the CFS condition were not associated with better catch trial performance for aligned faces, and, therefore, the composite effect in the CFS condition did not seem to result from weak access to bottom halves.

\section{General discussion}

In the present study, multiple experiments were conducted to investigate whether holistic face processing could occur when facial information was presented unconsciously. First, the composite effect, as measured by the complete design of the composite task, was found both in the CFS and monocular conditions, indicating that the processing of top facial halves was affected by bottom halves when presented in both conditions. Second, the composite effect in the monocular condition was stronger and more consistent than that in the CFS condition, which suggests that much of the composite effect for consciously presented faces was disrupted when the irrelevant facial parts were masked. Third, the composite effect in the CFS condition was only discovered when the data of more than one experiment were combined together, but not when each was analyzed separately, suggesting that the composite effect in the CFS condition could only be detected by paradigms with sufficient power. Altogether, these results revealed that when the bottom halves of faces were rendered invisible, they could still influence the processing of top halves. This finding suggests that holistic face processing can be executed without conscious awareness. These results extend previous findings and are the first to indicate that holistic face processing can occur 
regardless of whether observers have conscious access to the identity of inducing components.

Results of this study are different from those of Axelrod and Rees (2014), who failed to observe the interference of subliminal irrelevant parts of faces on the target parts (i.e., the eyes). One of the possible reasons for their null finding was that the statistical power of their design was not adequate, for example, because of their small sample sizes (15 and 18 participants for the two experiments respectively). This conjecture is supported by our findings that the composite effect in the CFS condition was only observed when enough participants were included. Another possible reason for the divergence between the results here and those of Axelrod and Rees (2014) was the utilization of different dependent variables. In this study, both sensitivity $d$ ' and correct response times were employed, and the composite effect in the CFS condition was mainly found for response times. Nevertheless, this dependent variable was not analyzed by Axelrod and Rees as they asked participants to maximize accuracy rather than asking them to respond as accurately and quickly as possible. Instead, the dependent variable they analyzed was accuracy, which was not reliable in their experimental designs in that the interference of irrelevant parts could not be dissociated from guessing or response bias.

The composite effect in the CFS condition was weaker than when all face information was displayed consciously, but was still present when data were pooled across experiments. What kinds of facial information influence the processing of the target halves, producing the composite effect in CFS condtions? Previous results showed that composite effects were stronger for faces with low spatial frequency compared with middle or high spatial frequency (Goffaux, 2009; Goffaux \& Rossion, 2006; but see Cheung, Richler, Palmeri, \& Gauthier, 2008). Additionally, there is evidence that low spatial frequency information is more easily processed subliminally. For example, De Gardelle and Kouider (2010) utilized a frame 
judgment task and discovered that the priming effects of low spatial frequency information were independent of conscious awareness, whereas the priming effects of high spatial frequency information were associated with the stimulus visibility. Similarly, Khalid et al. (2013) employed gender masking priming paradigms and found priming effects only for masked faces with low spatial frequency information, but not for masked faces with high spatial frequency information. Thus, there is evidence that it is the low spatial frequency information that was integrated into the holistic representation when face components were presented subliminally.

In this study, catch trials were also employed to assess whether the bottom facial halves could be identified during the composite face task. Results of catch trials interleaved in the composite tasks showed that participants were not always able to identify the subliminal bottom halves of study faces, even when they influenced the processing of top halves. The results do not support the view that composite effects observed in the CFS conditions of Experiment 1 might be due to participants' conscious perception of identity information from the bottom halves. In fact, even for the supraliminal composites showing clear composite effects, the bottom halves of study faces were still not always identifiable. In Experiments $2 \mathrm{~b}$, the bottom halves of aligned test faces were identified by participants in both monocular and CFS conditions but the performance appeared rather poor. Taken together, these catch trials suggest that whether participants have access to the identity information of bottom halves of test faces is not mediated by visual awareness. Because the bottom halves are irrelevant, participants are likely to disregard them, rendering them not consciously accessible. In this way, the bottom halves appear to be subject to inattentional or 
irrelevance blindness (e.g., Eitam, Shoval, \& Yeshurun, 2015; Eitam, Yeshurun, \& Hassan, 2013; Mack \& Rock, 1998). ${ }^{5}$

One interesting finding in this study was that the composite effect in the monocular condition was stronger than that in the CFS condition, even though the aligned bottom facial halves in catch trials were hardly identifiable in either task. These results imply that composite effects, even in the monocular condition, were not related to conscious processing according to Dehaene and colleagues (2006). Since the irrelevant bottom halves were not masked, not attended, and even hardly reportable, the processing of them in the monocular condition likely reflects preconscious processing, a process carrying information potentially for conscious access but temporally stored in a non-conscious buffer, while that in the CFS condition reflects subliminal processing, i.e., a condition of information inaccessibility (Dehaene et al., 2006). Critically, this preconscious and subliminal processing of bottom halves did influence the processing of top halves, as shown by the composite effects. Therefore, the composite effects in monocular and CFS conditions may reflect the preconscious and subliminal processing, respectively. The reduced composite effects in the CFS relative to monocular condition may show a lack of preconscious processing in that condition. This framework brings new insights to the understanding of face holistic processing, especially for faces presented consciously. We speculate that the composite effects measured by the modified complete composite task used in our study and the standard composite task are likely to relate to preconscious processing. By contrast, the complete

\footnotetext{
${ }^{5}$ We thank one anonymous reviewer for this suggestion.
} 
composite task with cues, where both top and bottom facial halves are attended at least for study faces, may show the influence of conscious processing.

It remains possible that participants might see the bottom halves of aligned test faces during composite tasks in the CFS condition of Experiment 2, but the probability should be quite small. A previous study found that image suppressions of CFS are quite strong and could last longer than three minutes with a mean of 56 seconds (Tsuchiya \& Koch, 2005), which was far longer than the duration used here, 300ms. Moreover, during the composite task, participants were instructed to focus on the top facial halves and there were few catch trials. With their attention being located on the top halves, suppressions for the bottom halves should be stronger. Furthermore, it is possible that participants mistakenly exploited the bottom halves of study faces, which were not covered by CFS, to complete some of the catch trials. When the bottom parts of study and test faces happened to be the same, participants would make correct responses. Critically, in the CFS condition, participants with higher accuracy in catch trials showed weaker composite effects. This evidence indicates that even if participants saw some of the bottom facial halves in the CFS condition, this weak conscious of bottom halves did not seem to contribute to the composite effects.

The performance of catch trials being above chance might suggest that face identity information could be processed unconsciously. This possibility stands in contrast to one previously reported result (Moradi et al., 2005), which did not observe an identity aftereffect when the adaptor faces were presented subliminally. Their observation of null results might be due to the use of an adaptation paradigm, since several other studies have found that the use of adaptation paradigms leads to a failure to observe unconscious face processing (Amihai et al., 2011; Pessoa, 2005; Tamietto \& de Gelder, 2010; Yang, Hong, \& Blake, 2010; but see Adams, Gray, Garner, \& Graf, 2010), while studies utilizing masking priming have shown that the facial information could be processed unconsciously (e.g., Finkbeiner \& 
Palermo, 2009; Khalid et al., 2013; Quek \& Finkbeiner, 2014). Considering the distinctive neural mechanisms underlying these two paradigms (Walther, Schweinberger, Kaiser, \& Kovács, 2013), the paradigm used here, which is more similar to masked priming, may be a more effective method to investigate unconscious face processing.

In our study, holistic processing was measured by the composite task, which reflects the influence of irrelevant parts on processing of target parts. This study provides the first evidence that the target facial halves were influenced by the other halves even when they were presented unconsciously, suggesting that holistic face processing can occur unconsciously. We also note that several studies have suggested that there are multiple constructs of holistic processing (e.g., Rezlescu et al., 2017; Richler et al., 2012; Rossion, 2013). For example, in addition to the interactions between facial parts used in this study, another facet of holistic processing is that facial parts cannot be extracted from a whole face representation (Tanaka \& Farah, 1993). Further efforts are needed to explore the role of conscious awareness in different constructs of holistic processing. 


\section{References}

Adams, W. J., Gray, K. L. H., Garner, M., \& Graf, E. W. (2010). High-Level Face Adaptation Without Awareness. Psychological Science, 21(2), 205-210. https://doi.org/10.1177/0956797609359508

Amihai, I., Deouell, L., \& Bentin, S. (2011). Conscious awareness is necessary for processing race and gender information from faces. Consciousness and Cognition, 20(2), 269-279. https://doi.org/10.1016/j.concog.2010.08.004

Amishav, R., \& Kimchi, R. (2010). Perceptual integrality of componential and configural information in faces. Psychonomic Bulletin \& Review, 17(5), 743-748. https://doi.org/10.3758/PBR.17.5.743

Axelrod, V., Bar, M., \& Rees, G. (2015). Exploring the unconscious using faces. Trends in Cognitive Sciences, 19(1), 35-45. https://doi.org/10.1016/j.tics.2014.11.003

Axelrod, V., \& Rees, G. (2014). Conscious awareness is required for holistic face processing. Consciousness and Cognition, 27(1), 233-245. https://doi.org/10.1016/j.concog.2014.05.004

Brainard, D. H. (1997). The Psychophysics Toolbox. Spatial Vision, 10(4), 433-436. https://doi.org/10.1163/156856897X00357

Busigny, T., Van Belle, G., Jemel, B., Hosein, A., Joubert, S., \& Rossion, B. (2014). Facespecific impairment in holistic perception following focal lesion of the right anterior temporal lobe. Neuropsychologia, 56(1), 312-333. https://doi.org/10.1016/j.neuropsychologia.2014.01.018

Cheung, O. S., Richler, J. J., Palmeri, T. J., \& Gauthier, I. (2008). Revisiting the role of 
spatial frequencies in the holistic processing of faces. Journal of Experimental Psychology: Human Perception and Performance, 34(6), 1327-1336. https://doi.org/10.1037/a0011752

Chua, K.-W., Richler, J. J., \& Gauthier, I. (2014). Becoming a Lunari or Taiyo expert: Learned attention to parts drives holistic processing of faces. Journal of Experimental Psychology: Human Perception and Performance, 40(3), 1174-1182. https://doi.org/10.1037/a0035895

de Gardelle, V., \& Kouider, S. (2010). How Spatial Frequencies and Visual Awareness Interact During Face Processing. Psychological Science, 21(1), 58-66. https://doi.org/10.1177/0956797609354064

Dehaene, S., Changeux, J.-P., Naccache, L., Sackur, J., \& Sergent, C. (2006). Conscious, preconscious, and subliminal processing: a testable taxonomy. Trends in Cognitive Sciences, 10(5), 204-211. https://doi.org/10.1016/j.tics.2006.03.007

Duchaine, B., \& Nakayama, K. (2006). The Cambridge Face Memory Test: Results for neurologically intact individuals and an investigation of its validity using inverted face stimuli and prosopagnosic participants. Neuropsychologia, 44(4), 576-585. https://doi.org/10.1016/j.neuropsychologia.2005.07.001

Eitam, B., Shoval, R., \& Yeshurun, Y. (2015). Seeing without knowing: task relevance dissociates between visual awareness and recognition. Annals of the New York Academy of Sciences, 1339(1), 125-137. https://doi.org/10.1111/nyas.12673

Eitam, B., Yeshurun, Y., \& Hassan, K. (2013). Blinded by irrelevance: Pure irrelevance induced "blindness". Journal of Experimental Psychology: Human Perception and Performance, 39(3), 611-615. https://doi.org/10.1037/a0032269 
Finkbeiner, M., \& Palermo, R. (2009). The Role of Spatial Attention in Nonconscious Processing. Psychological Science, 20(1), 42-51. https://doi.org/10.1111/j.14679280.2008.02256.x

Ge, L., Zhang, H., Wang, Z., Quinn, P. C., Pascalis, O., Kelly, D. J., ... Lee, K. (2009). Two faces of the other-race effect: Recognition and categorisation of Caucasian and Chinese faces. Perception, 38(8), 1199-1210. https://doi.org/10.1068/p6136

Goffaux, V. (2009). Spatial interactions in upright and inverted faces: Re-exploration of spatial scale influence. Vision Research, 49(7), 774-781. https://doi.org/10.1016/j.visres.2009.02.009

Goffaux, V., \& Rossion, B. (2006). Faces are "spatial"--holistic face perception is supported by low spatial frequencies. Journal of Experimental Psychology: Human Perception and Performance, 32(4), 1023-1039. https://doi.org/10.1037/0096-1523.32.4.1023

Hayward, W. G., Crookes, K., Chu, M. H., Favelle, S. K., \& Rhodes, G. (2016). Holistic processing of face configurations and components. Journal of Experimental Psychology: Human Perception and Performance, 42(10), 1482-1489. https://doi.org/10.1037/xhp0000246

Hayward, W. G., Crookes, K., \& Rhodes, G. (2013). The other-race effect: Holistic coding differences and beyond. Visual Cognition, 21(9-10), 1-24. https://doi.org/10.1080/13506285.2013.824530

Henson, R. N. N., Mouchlianitis, E., Matthews, W. J. J., \& Kouider, S. (2008). Electrophysiological correlates of masked face priming. NeuroImage, 40(2), 884-895. https://doi.org/10.1016/j.neuroimage.2007.12.003

Hole, G. J. (1994). Configurational Factors in the Perception of Unfamiliar Faces. 
Perception, 23(1), 65-74. https://doi.org/10.1068/p230065

Jacques, C., \& Rossion, B. (2009). The initial representation of individual faces in the right occipito-temporal cortex is holistic: Electrophysiological evidence from the composite face illusion. Journal of Vision, 9(6), 1-16. https://doi.org/10.1167/9.6.8.Introduction

Jacques, C., \& Rossion, B. (2010). Misaligning face halves increases and delays the N170 specifically for upright faces: implications for the nature of early face representations. Brain Research, 1318, 96-109. https://doi.org/10.1016/j.brainres.2009.12.070

Jiang, F., Blanz, V., \& Rossion, B. (2011). Holistic processing of shape cues in face identification: Evidence from face inversion, composite faces, and acquired prosopagnosia. Visual Cognition, 19(8), 1003-1034. https://doi.org/10.1080/13506285.2011.604360

Jiang, Y., \& He, S. (2006). Cortical responses to invisible faces: dissociating subsystems for facial-information processing. Current Biology, 16(20), 2023-2029. https://doi.org/10.1016/j.cub.2006.08.084

Jiang, Y., Shannon, R. W., Vizueta, N., Bernat, E. M., Patrick, C. J., \& He, S. (2009). Dynamics of processing invisible faces in the brain: Automatic neural encoding of facial expression information. NeuroImage, 44(3), 1171-1177. https://doi.org/10.1016/j.neuroimage.2008.09.038

Khalid, S., Finkbeiner, M., König, P., \& Ansorge, U. (2013). Subcortical human face processing? Evidence from masked priming. Journal of Experimental Psychology: Human Perception and Performance, 39(4), 989-1002. https://doi.org/10.1037/a0030867

Kiss, M., \& Eimer, M. (2008). ERPs reveal subliminal processing of fearful faces. 
Psychophysiology, 45(2), 318-326. https://doi.org/10.1111/j.1469-8986.2007.00634.x

Kouider, S., Eger, E., Dolan, R., \& Henson, R. N. (2009). Activity in face-responsive brain regions is modulated by invisible, attended faces: Evidence from masked priming. Cerebral Cortex, 19(1), 13-23. https://doi.org/10.1093/cercor/bhn048

Laguesse, R., \& Rossion, B. (2013). Face perception is whole or none: disentangling the role of spatial contiguity and interfeature distances in the composite face illusion. Perception, 42(10), 1013-1026. https://doi.org/10.1068/p7534

Lenth, R. (2019). emmeans: Estimated Marginal Means, aka Least-Squares Means. Retrieved from https://cran.r-project.org/package=emmeans

Mack, A., \& Rock, I. (1998). Inattentional Blindness. https://doi.org/10.7551/mitpress/3707.001.0001

Maurer, D., Grand, R. Le, \& Mondloch, C. J. (2002). The many faces of configural processing. Trends in Cognitive Sciences, 6(6), 255-260. Retrieved from http://www.ncbi.nlm.nih.gov/pubmed/12039607

Meinhardt-Injac, B., Persike, M., \& Meinhardt, G. (2010). The time course of face matching by internal and external features: Effects of context and inversion. Vision Research, 50(16), 1598-1611. https://doi.org/10.1016/j.visres.2010.05.018

Meinhardt-Injac, B., Persike, M., \& Meinhardt, G. (2014). Integration of internal and external facial features in 8- to 10-year-old children and adults. Acta Psychologica, 149, 96-105. https://doi.org/10.1016/j.actpsy.2014.03.008

Miles, W. R. (1930). Ocular Dominance in Human Adults. The Journal of General Psychology, 3(3), 412-430. https://doi.org/10.1080/00221309.1930.9918218 
Moradi, F., Koch, C., \& Shimojo, S. (2005). Face adaptation depends on seeing the face. Neuron, 45(1), 169-175. https://doi.org/10.1016/j.neuron.2004.12.018

Pessoa, L. (2005). To what extent are emotional visual stimuli processed without attention and awareness? Current Opinion in Neurobiology, 15(2), 188-196. https://doi.org/10.1016/j.conb.2005.03.002

Posner, M. I., \& Snyder, C. R. R. (1975). Attention and cognitive control. In R. L. Solso (Ed.), Information Processing and Cognition: The Loyola Symposium. Lawrence Erlbaum.

Quek, G. L., \& Finkbeiner, M. (2014). Face-sex categorization is better above fixation than below: Evidence from the reach-to-touch paradigm. Cognitive, Affective, \& Behavioral Neuroscience, 14(4), 1407-1419. https://doi.org/10.3758/s13415-014-0282-y

R Core Team. (2018). R: A language and environment for statistical computing. Retrieved from https://www.r-project.org/

Retter, T. L., \& Rossion, B. (2015). Global shape information increases but color information decreases the composite face effect. Perception, 44(5), 511-528. https://doi.org/10.1068/p7826

Rezlescu, C., Susilo, T., Wilmer, J. B., \& Caramazza, A. (2017). The inversion, part-whole, and composite effects reflect distinct perceptual mechanisms with varied relationships to face recognition. Journal of Experimental Psychology: Human Perception and Performance, 43(12), 1961-1973. https://doi.org/10.1037/xhp0000400

Richler, J. J., Cheung, O. S., \& Gauthier, I. (2011). Beliefs alter holistic face processing ... if response bias is not taken into account. Journal of Vision, 11(13), 17-17.

https://doi.org/10.1167/11.13.17 
Richler, J. J., \& Gauthier, I. (2013). When intuition fails to align with data: A reply to Rossion (2013). Visual Cognition, 21(2), 254-276.

https://doi.org/10.1080/13506285.2013.796035

Richler, J. J., \& Gauthier, I. (2014). A meta-analysis and review of holistic face processing. Psychological Bulletin, 140(5), 1281-1302. https://doi.org/10.1037/a0037004

Richler, J. J., Palmeri, T. J., \& Gauthier, I. (2012). Meanings, Mechanisms, and Measures of Holistic Processing. Frontiers in Psychology, 3, 553.

https://doi.org/10.3389/fpsyg.2012.00553

Richler, J. J., Palmeri, T. J., \& Gauthier, I. (2015). Holistic processing does not require configural variability. Psychonomic Bulletin \& Review, 22(4), 974-979. https://doi.org/10.3758/s13423-014-0756-5

Richler, J. J., Wong, Y. K., \& Gauthier, I. (2011). Perceptual Expertise as a Shift from Strategic Interference to Automatic Holistic Processing. Current Directions in Psychological Science, 20(2), 129-134. https://doi.org/10.1177/0963721411402472

Rosenthal, G., Levakov, G., \& Avidan, G. (2018). Holistic face representation is highly orientation-specific. Psychonomic Bulletin \& Review, 25(4), 1351-1357. https://doi.org/10.3758/s13423-017-1381-x

Rossion, B. (2008). Picture-plane inversion leads to qualitative changes of face perception. Acta Psychologica, 128(2), 274-289. https://doi.org/10.1016/j.actpsy.2008.02.003

Rossion, B. (2013). The composite face illusion: A whole window into our understanding of holistic face perception. Visual Cognition, 21(2), 139-253. https://doi.org/10.1080/13506285.2013.772929 
Rossion, B. (2018). Humans Are Visual Experts at Unfamiliar Face Recognition. Trends in Cognitive Sciences, 22(6), 471-472. https://doi.org/10.1016/j.tics.2018.03.002

Rossion, B., \& Michel, C. (2018). Normative accuracy and response time data for the computerized Benton Facial Recognition Test (BFRT-c). Behavior Research Methods, 50(6), 2442-2460. https://doi.org/10.3758/s13428-018-1023-x

Rossion, B., \& Retter, T. L. (2015). Holistic face perception: Mind the gap! Visual Cognition, 23(3), 379-398. https://doi.org/10.1080/13506285.2014.1001472

RStudio Team. (2019). RStudio: Integrated Development for R. RStudio. Retrieved from http://www.rstudio.com/.

Sato, W., Okada, T., \& Toichi, M. (2007). Attentional shift by gaze is triggered without awareness. Experimental Brain Research, 183(1), 87-94. https://doi.org/10.1007/s00221-007-1025-x

Singmann, H., Bolker, B. M., Westfall, J., \& Aust, F. (2019). afex: Analysis of Factorial Experiments. Retrieved from https://cran.r-project.org/package=afex

Stein, T., Hebart, M. N., \& Sterzer, P. (2011). Breaking Continuous Flash Suppression: A New Measure of Unconscious Processing during Interocular Suppression? Frontiers in Human Neuroscience, 5(December), 1-17. https://doi.org/10.3389/fnhum.2011.00167

Stein, T., Peelen, M. V., \& Sterzer, P. (2012). Eye gaze adaptation under interocular suppression. Journal of Vision, 12(7), 1-1. https://doi.org/10.1167/12.7.1

Stein, T., \& Sterzer, P. (2011). High-level face shape adaptation depends on visual awareness: Evidence from continuous flash suppression. Journal of Vision, 11(8), 5-5. https://doi.org/10.1167/11.8.5 
Tamietto, M., \& de Gelder, B. (2010). Neural bases of the non-conscious perception of emotional signals. Nature Reviews Neuroscience, 11(10), 697-709. https://doi.org/10.1038/nrn2889

Tanaka, J. W., \& Farah, M. J. (1993). Parts and Wholes in Face Recognition. The Quarterly Journal of Experimental Psychology Section A, 46(2), 225-245. https://doi.org/10.1080/14640749308401045

Tanaka, J. W., \& Sengco, J. A. (1997). Features and their configuration in face recognition. Memory \& Cognition, 25(5), 583-592. https://doi.org/10.3758/BF03211301

Tanaka, J. W., \& Simonyi, D. (2016). The "parts and wholes" of face recognition: A review of the literature. The Quarterly Journal of Experimental Psychology, 69(10), 18761889. https://doi.org/10.1080/17470218.2016.1146780

The jamovi project. (2019). jamovi. Retrieved from https://www.jamovi.org

Tsuchiya, N., \& Koch, C. (2005). Continuous flash suppression reduces negative afterimages. Nature Neuroscience, 8(8), 1096-1101. https://doi.org/10.1038/nn1500

Walther, C., Schweinberger, S. R., Kaiser, D., \& Kovács, G. (2013). Neural correlates of priming and adaptation in familiar face perception. Cortex, 49(7), 1963-1977. https://doi.org/10.1016/j.cortex.2012.08.012

Wang, Z., Quinn, P. C., Jin, H., Sun, Y.-H. P., Tanaka, J. W., Pascalis, O., \& Lee, K. (2019). A regional composite-face effect for species-specific recognition: Upper and lower halves play different roles in holistic processing of monkey faces. Vision Research, 157, 89-96. https://doi.org/10.1016/j.visres.2018.03.004

Wickham, H. (2017). tidyverse: Easily Install and Load the “Tidyverse.” Retrieved from 
https://cran.r-project.org/package=tidyverse

Willenbockel, V., Sadr, J., Fiset, D., Horne, G. O., Gosselin, F., \& Tanaka, J. W. (2010). Controlling low-level image properties: The SHINE toolbox. Behavior Research Methods, 42(3), 671-684. https://doi.org/10.3758/BRM.42.3.671

Williams, M. A., Morris, A. P., McGlone, F., Abbott, D. F., \& Mattingley, J. B. (2004). Amygdala Responses to Fearful and Happy Facial Expressions under Conditions of Binocular Suppression. Journal of Neuroscience, 24(12), 2898-2904. https://doi.org/10.1523/JNEUROSCI.4977-03.2004

Yang, E., Hong, S.-W., \& Blake, R. (2010). Adaptation aftereffects to facial expressions suppressed from visual awareness. Journal of Vision, 10(12), 24-24. https://doi.org/10.1167/10.12.24

Yokoyama, T., Noguchi, Y., \& Kita, S. (2013). Unconscious processing of direct gaze: Evidence from an ERP study. Neuropsychologia, 51(7), 1161-1168. https://doi.org/10.1016/j.neuropsychologia.2013.04.002

Young, A. W., \& Burton, A. M. (2018a). Are We Face Experts? Trends in Cognitive Sciences, 22(2), 100-110. https://doi.org/10.1016/j.tics.2017.11.007

Young, A. W., \& Burton, A. M. (2018b). What We See in Unfamiliar Faces: A Response to Rossion. Trends in Cognitive Sciences, 22(6), 472-473. https://doi.org/10.1016/j.tics.2018.03.008

Young, A. W., Hellawell, D., \& Hay, D. C. (1987). Configurational information in face perception. Perception, 16(6), 747-759. https://doi.org/10.1068/p160747 


\section{Supplementary materials}

\section{S1. Results of Experiment 1a}

\section{S1.1. Sensitivity $d^{\prime}$}

Separate 2 (Congruency: congruent vs. incongruent) $\times 2$ (Alignment: aligned vs misaligned) repeated-measures ANOVAs were conducted on the sensitivity $d$ ' for each task in Experiment 1a (Figure S1a). Results showed that the interaction between Congruency and Alignment in the CFS viewing condition was not significant $(F<.07)$. By contrast, the interaction in the monocular viewing condition was significant, $F(1,19)=5.69, M S E=.16, p$ $<.05$, partial $\eta^{2}=.23$. Simple effects analyses showed better performance for congruent trials $(M=2.78,95 \% C I[2.46,3.10])$ compared to incongruent trials $(M=2.17,95 \% C I[1.85$, $2.49])$ in the aligned condition $(p<.001)$ and no significant differences in the misaligned conditions (congruent: $M=2.68,95 \% C I[2.35,3.00]$; incongruent: $M=2.48,95 \% C I[2.16$, 2.81]; $p>.27$ ). These results suggest that the composite effect was found in the monocular, but not the CFS viewing condition.

\section{S1.2. Correct Response Times}

Separate 2 (Congruency: congruent vs. incongruent) $\times 2$ (Alignment: aligned vs

misaligned) repeated-measures ANOVAs were conducted on the correct response times for each task (Figure S1b). For the composite face task with the CFS, the interaction was significant, $F(1,19)=5.00, M S E=285, p<.05$, partial $\eta^{2}=.21$. However, this significant interaction cannot be taken as the evidence of composite effect (see main texts). For the 

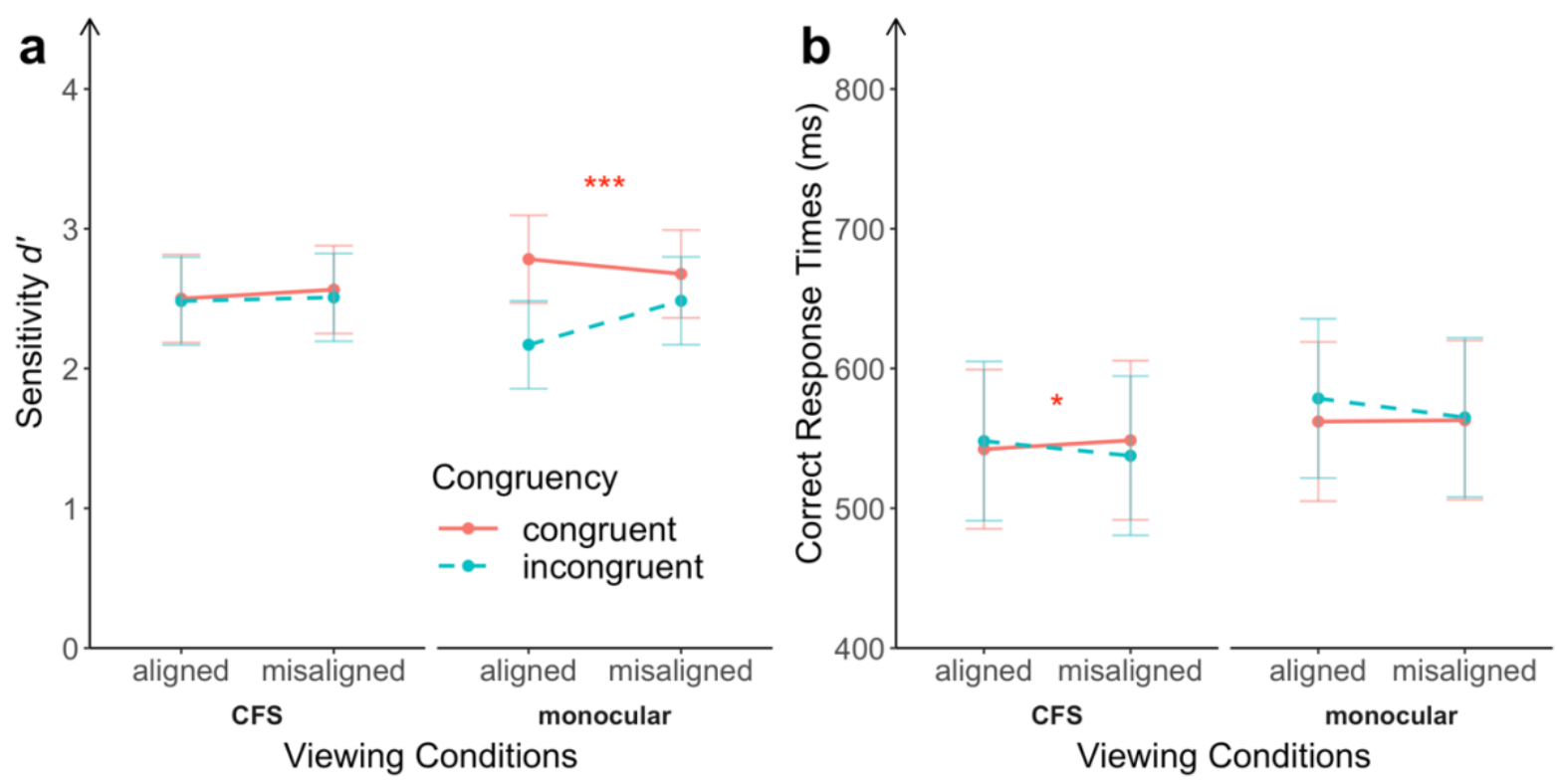

Figure S1. (a) Sensitivity $d$ ' and (b) correct response times as a function of Congruency and Alignment in the CFS and monocular viewing conditions for Experiment 1a. Error bars represent 95\% confidence intervals. Asterisks indicate significant interaction between Congruency and Alignment $(*, p<.05 ; * * *, p$ $<.001)$.

monocular version of the composite task, the interaction did not reach significance, $F(1,19)$ $=1.64, M S E=646, p>.21$, partial $\eta^{2}=.08$.

\section{S2. Results of Experiment 1b}

\section{S2.1. Sensitivity $d^{\prime}$}

Two separate 2 (Congruency: congruent vs. incongruent) $\times 2$ (Alignment: aligned vs. misaligned) repeated-measures ANOVAs were conducted on the sensitivity $d$ ' of the two different viewing conditions (Figure S2a). Results of the CFS task showed the interaction between Congruency and Alignment was not significant $(F<.05)$, indicating that no composite effect was observed for the sensitivity $d^{\prime}$ in the CFS composite task. On the other hand, the interaction between Congruency and Alignment was significant, $F(1,29)=16.28$, $M S E=.15, p<.001$, partial $\eta^{2}=.36$. Simple effects analyses showed better performance for congruent composites $(M=2.89,95 \% C I[2.593 .18])$ compared with incongruent composites in the aligned condition $(M=2.25,95 \% C I[1.952 .55], p<.001)$ and, yet, no significant 

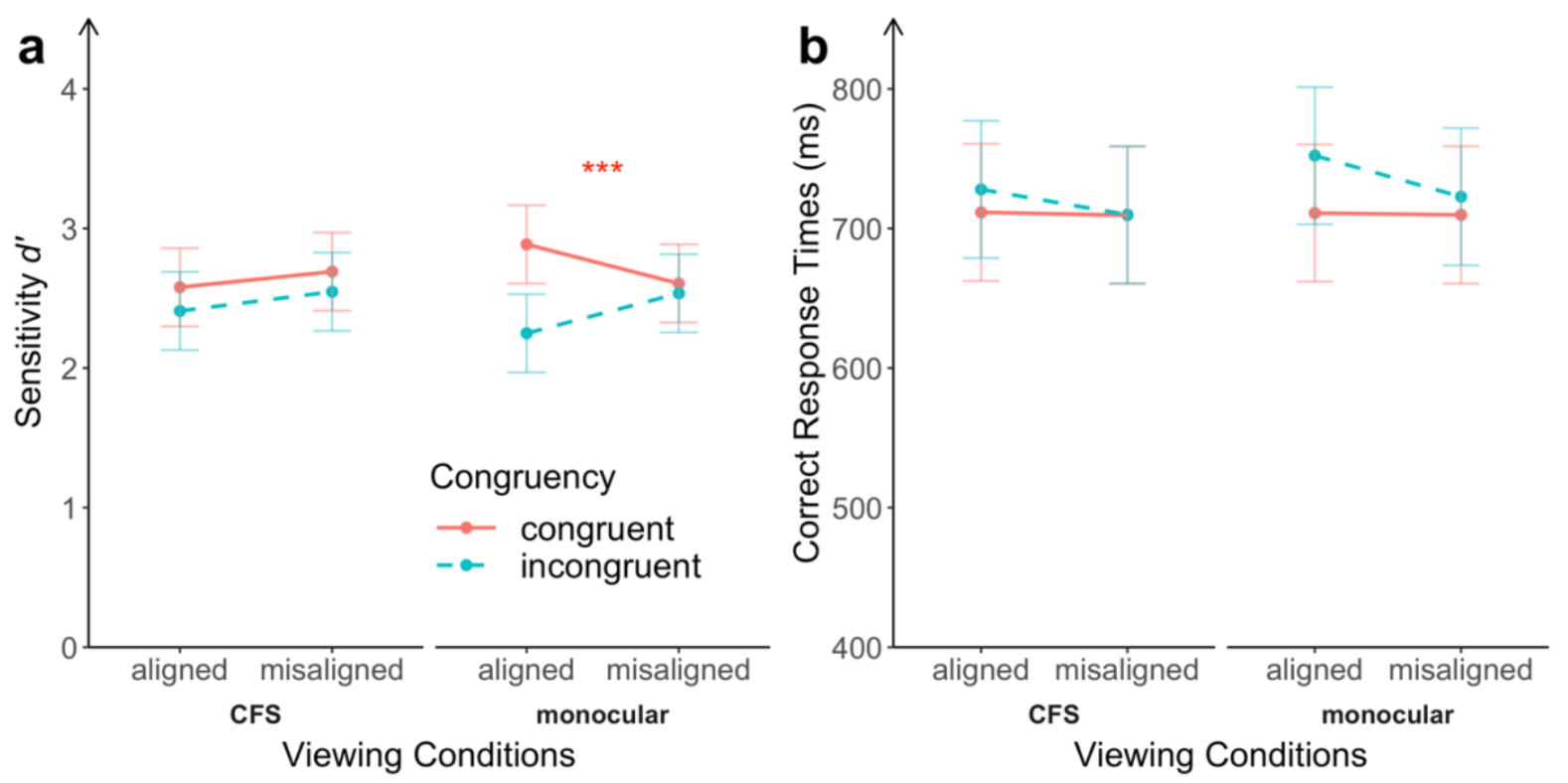

Figure S1. (a) Sensitivity $d$ ' and (b) correct response times as a function of Congruency and Alignment in the CFS and monocular viewing conditions for Experiment 1b. Error bars represent $95 \%$ confidence intervals. Asterisks indicate significant interaction between Congruency and Alignment $\left({ }^{* * *}, p<.001\right)$.

differences in the misaligned conditions (congruent: $M=2.61,95 \% C I$ [2.31 2.90]; incongruent: $M=2.54,95 \% C I[2.242 .83], p>.99)$, suggesting that the composite effect was observed for the sensitivity $d$ ' in the monocular condition.

\section{S2.2. Correct response times}

Separate 2 (Congruency: congruent vs. incongruent) $\times 2$ (Alignment: aligned vs. misaligned) repeated-measures ANOVAs were conducted on the correct response times for the two composite tasks (Figure S2b). For the CFS composite task, the interaction ${ }^{1}$ between Congruency and Alignment did not reach significance, $F(1,29)=2.09, M S E=926, p=.16$, partial $\eta^{2}=.07$. These results suggested that no composite effect was found for the correct response times in the CFS composite task. For the monocular composite task, the critical

\footnotetext{
${ }^{1}$ In the standard design analyses, response times of aligned trials were longer than those of misaligned
} trials in the CFS condition $(t(57.92)=2.39, p<.05)$, showing the composite effect in the CFS condition. 
interaction $^{2}$ between them was marginally significant, $F(1,29)=3.12, M S E=1895, p=.088$, partial $\eta^{2}=.10$. Simple effects analyses showed shorter correct response times for congruent trials $(M=711,95 \% C I[659763])$ than incongruent trials in the aligned condition $(M=752$, $95 \% C I[701804], p<.001)$ and no differences in the misaligned condition (congruent: $M=$ $710,95 \% C I[658761]$; incongruent: $M=723,95 \% C I[671774], p>.47)$, indicating the composite effect was found for the correct response times in the monocular composite face task.

\section{S3. Results of Experiment 2a}

\section{S3.1. Sensitivity $d^{\prime}$}

Two separate 2 (Congruency: congruent vs. incongruent) $\times 2$ (Alignment: aligned vs. misaligned) repeated-measures ANOVAs were conducted on the sensitivity $d$ ' of the two composite face tasks (Figure S3a). No significant results were found for the interaction between Congruency and Alignment in the CFS task, $F(1,19)=1.01, M S E=.17, p>.32$, partial $\eta^{2}=.05$. This non-significant interaction did not provide evidence that composite effects were found for sensitivity in the CFS condition, even though only the test faces were covered. Results of the monocular task showed the interaction between Congruency and Alignment reached significance, $F(1,19)=4.83, M S E=.29, p<.05$, partial $\eta^{2}=.20$. Simple effects analyses showed the performance of congruent trials $(M=2.72,95 \% C I[2.423 .03])$ were better than incongruent trials in the aligned condition $(M=1.98,95 \% C I$ [1.68 2.29], $p$

\footnotetext{
${ }^{2}$ In the standard design analyses, response times of aligned trials were longer than those of misaligned trials in the monocular condition $(t(57.92)=4.59, p<.001)$, displaying the composite effect in the monocular condition.
} 

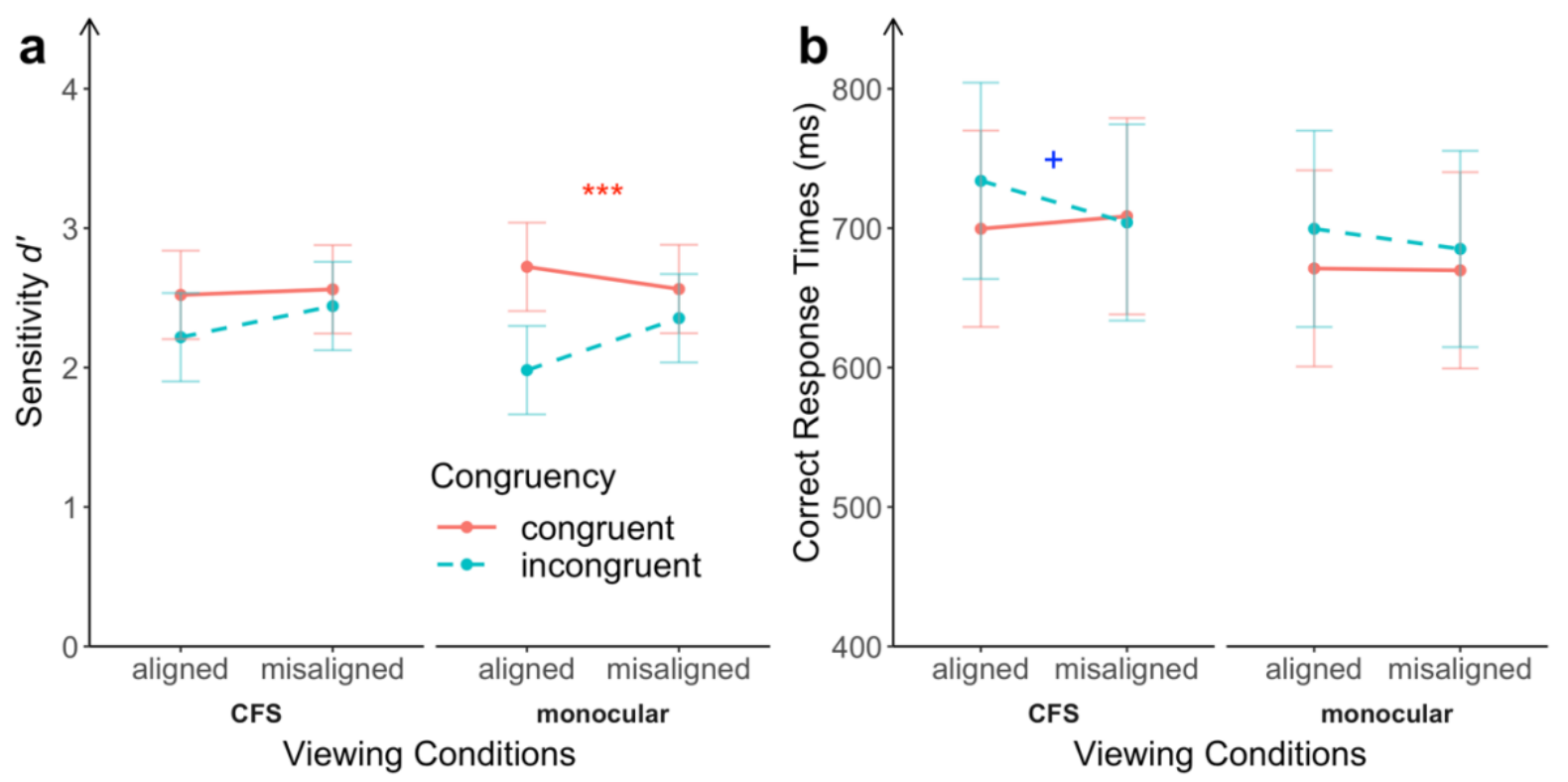

Figure S1. (a) Sensitivity $d$ ' and (b) correct response times as a function of Congruency and Alignment in the CFS and monocular viewing conditions for Experiment 2a. Error bars represent $95 \%$ confidence intervals. Asterisks indicate significant interaction between Congruency and Alignment $(+, p=.056 ; * * *$, $p<.001)$.

$<.001$ ) and no differences were found in the misaligned conditions (congruent: $M=2.56$, 95\% $C I$ [2.26 2.87]; incongruent: $M=2.35,95 \% C I$ [2.05 2.66], $p>.32)$. These results verify the finding of a composite effect for the sensitivity $d$ ' in the monocular condition.

\section{S3.2. Correct response times}

Separate 2 (Congruency: congruent vs. incongruent) $\times 2$ (Alignment: aligned vs . misaligned) repeated-measures ANOVA were computed on the correct response times for the two composite face tasks (Figure S3b). For the CFS composite task, the critical interaction between Congruency and Alignment reached marginal significance ${ }^{3}, F(1,19)=4.16, M S E=$ $1813, p=.056$, partial $\eta^{2}=.18$. Simple effects analyses showed whereas the response times

\footnotetext{
${ }^{3}$ In the standard design analyses, response times of aligned trials were longer than those of misaligned
} trials in the CFS condition $(t(37.99)=2.87, p<.05)$, showing the composite effect in the CFS condition. 
of congruent trials $(M=700,95 \% C I[620779])$ were shorter than those of incongruent trials $(M=734,95 \% C I[654813])$ in the aligned condition $(p<.05)$, no significant results were found for the misaligned condition (congruent: $M=709,95 \% C I$ [629 788]; incongruent: ( $M$ $=704,95 \%$ CI [625 784], $\mathrm{p}>.99)$. These results suggest that the composite effect was found for the response times in the CFS condition. For the monocular composite task, nonsignificant results were found for the interaction, $F(1,19)=1.41, M S E=609, p=.25$, partial $\eta^{2}=.07$, failing to support that composite effects were found for correct response times in the monocular condition.

\section{S4. Results of Experiment 2b}

\section{S4.1. Sensitivity $d^{\prime}$}

Two separate 2 (Congruency: congruent vs. incongruent) $\times 2$ (Alignment: aligned vs. misaligned) repeated-measures ANOVAs were conducted on the sensitivity $d$ ' of the two composite face tasks (Figure S4a). Results of the CFS task showed the interaction between Congruency and Alignment was not significant, $F(1,29)=1.98, M S E=.23, p>.17$, partial $\eta^{2}=.06$. This result suggests that no composite effect was found for the sensitivity in the CFS composite task. On the other hand, the results of the monocular task showed the interaction between Congruency and Alignment was significant, $F(1,29)=40.38, M S E=.13$, $p<.001$, partial $\eta^{2}=.58$. Simple effects analyses showed better performance for congruent composites $(M=3.13,95 \% C I[2.863 .40])$ compared with incongruent composites in the aligned condition $(M=2.25,95 \% C I[1.982 .52], p<.001)$ and, yet, no differences in the misaligned conditions (congruent: $M=2.92,95 \% C I$ [2.65 3.19]; incongruent: $M=2.89$, $95 \% C I[2.623 .16], p>.99)$, showing the composite effect for the sensitivity in the monocular composite task. 

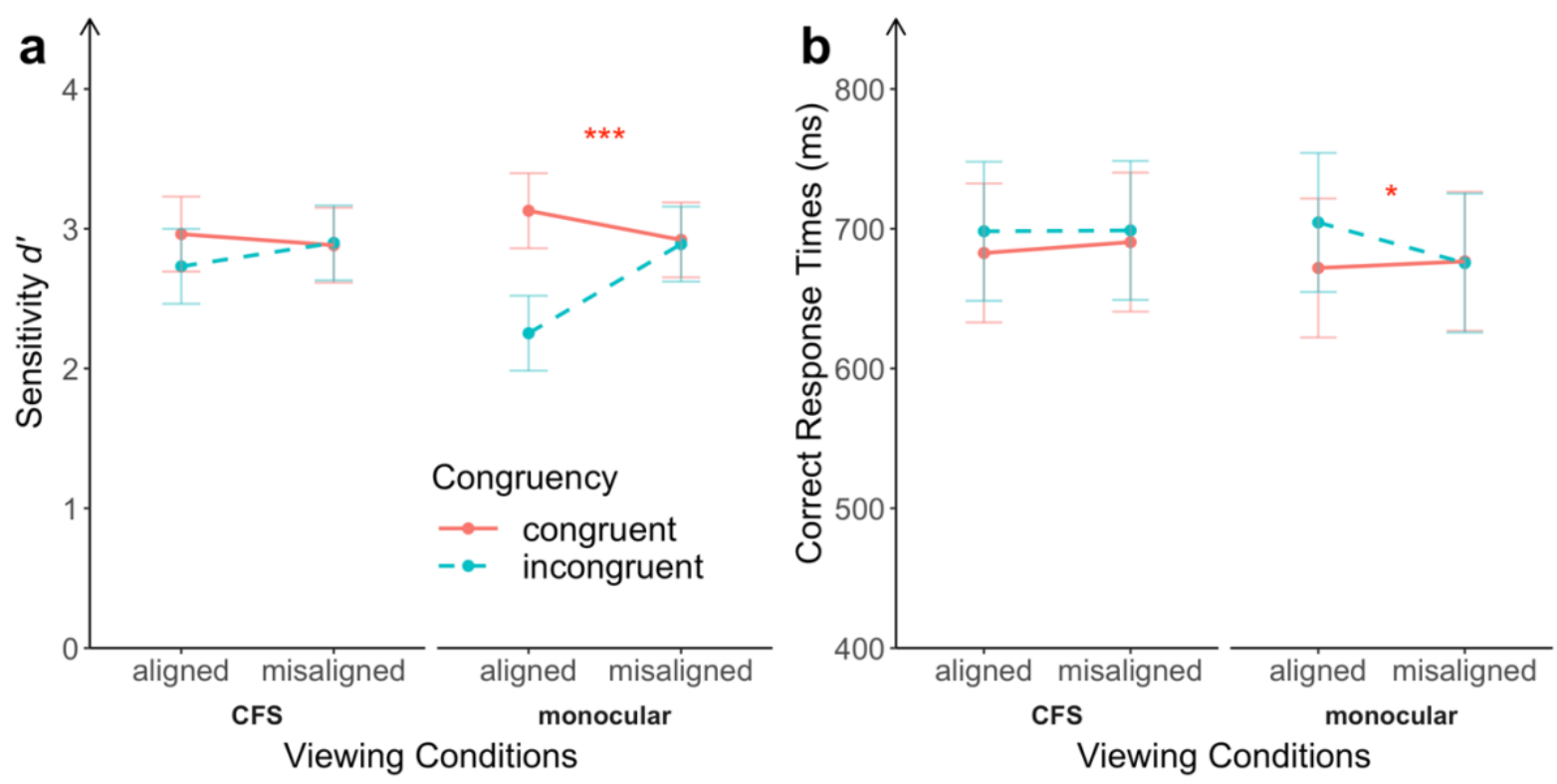

Figure S1. (a) Sensitivity $d$ ' and (b) correct response times as a function of Congruency and Alignment in the CFS and monocular viewing conditions for Experiment 2 b. Error bars represent $95 \%$ confidence intervals. Asterisks indicate significant interaction between Congruency and Alignment $\left({ }^{*}, p<.05 ; * * *, p\right.$ $<.001)$.

\section{S4.2. Correct response times}

Separate 2 (Congruency: congruent vs. incongruent) $\times 2$ (Alignment: aligned vs.

misaligned) repeated-measure ANOVAs on the correct response times for the two composite face tasks (Figure S4b). For the CFS composite task, the interaction between Congruency and Alignment were not significant $(F<.46)$, suggesting that no composite effect was observed for the correct response times in the CFS composite task. For the monocular composite task, the interaction was significant, $F(1,29)=6.73, M S E=1895, p<.05$, partial $\eta^{2}=.19$. Simple effects analysis showed shorter correct response times for congruent trials $(M=672,95 \% C I$ [620 724]) than incongruent trials in the aligned condition $(M=705,95 \% C I[653756], p$ $<.01$ ) and no differences in the misaligned condition (congruent: $M=677,95 \% C I[625$ 728]; incongruent: $M=675,95 \% C I[624727], p>.99)$, indicating that the composite effect was found for the correct response times in the monocular composite face task. 